\title{
Research Paper: \\ Mediating Role of Emotion Regulation in the Relationship of Metacognitive Beliefs and Attachment Styles With Risky Behaviors in Children of Iran-Iraq War Veterans With Psychiatric Disorders
}

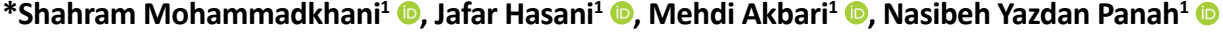

1. Department of Clinical Psychology, Faculty of Psychology and Education, Kharazmi University, Tehran, Iran

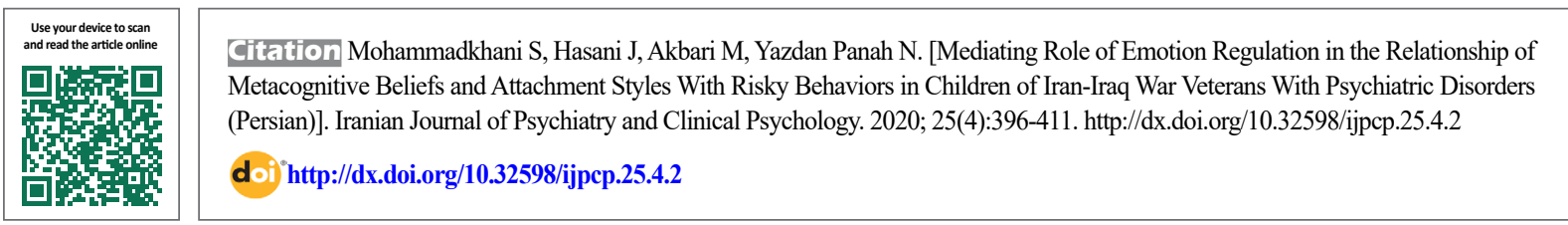

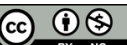

Received: 13 Nov 2018 Accepted: 19 Aug 2019 Available Online: 01 Jan 2020

Key words: Emotion regulation Attachment style, Metacognition, Risky behavior, Adolescent, War veterans

\section{A B STRACT}

Objectives Risk behaviors among adolescents are a major concern for mental and social health. The purpose of this study was to investigate the mediating role of adaptive and maladaptive emotion regulation strategies in the relationship of metacognitive beliefs and attachment styles with risky behaviors among adolescent children of war veterans with psychiatric disorders.

Methods This is a descriptive correlational study conducted on 243 adolescent children of Iraq-Iran war veterans suffering from psychiatric disorders who were selected using a convenience sampling method. They responded to Cognitive Emotion Regulation Questionnaire (Short form), Revised Adult attachment Scale, Metacognitions Questionnaire for Children, and Risky Behaviors Scale.

Results emotion regulation strategies mediated the relationship of metacognitive beliefs and attachment styles with risky behaviors. There was no significant relationship between adaptive strategies of emotion regulation and risky behaviors, and between metacognitive beliefs adaptive strategies of emotion regulation $(\mathrm{P}>0.001)$, but the relationship between metacognitive beliefs and maladaptive strategies was significant. All attachment styles had a direct and significant correlation with both adaptive and maladaptive strategies of emotion regulation. The presented model could explain $14 \%$ of the risky behaviors in subjects.

Conclusion Metacognitive beliefs and attachment styles are directly and indirectly related to emotion regulation strategies and risky behaviors of the children of war veterans. These results can be used for designing the preventive programs for this group.

\section{Extended Abstract}

\section{Introduction}

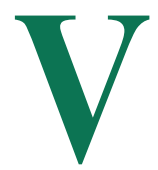

arious studies have shown that post-traumatic symptoms affect veterans' ability to play parental role [2]. The relationship between the injured veteran and his/her child is often characterized by conflict, control, excessive proximity and excessive protection. This, in turn, may lead to various psychiatric symptoms among their children, a phenomenon known as "secondary harm" [2]. Evidence of the transmission of damage from the parents to the second generation can be found in literature. One of the important studies was conducted on the children of Holocaust survivors who were reported to often have problems such as individuality, identity, repeated parental pathology, aggression, excessive guilt, depression and social isolation

* Corresponding Author:

Shahram Mohammadkhani, PhD.

Address: Department of Clinical Psychology, Faculty of Psychology and Education, Kharazmi University Clinical Psychology Department, Tehran, Iran Tel: +98 (21) 88356736

E-mail: mohammadkhani@khu.ac.ir 
[3]. On the other hand, adolescence is an important developmental period associated with the process of identity formation [4]. A part of the developmental process of identity formation is risk-taking which may appear as unhealthy sexual behavior, alcohol consumption, smoking, substance abuse, running away from home/school, and aggressive behaviors [5-7]. Hence, one of the mental health problems of adolescent children of war veterans can be risky behaviors.

In Iran, as one of the countries with war casualties, most of studies have focused on the effects of Iran-Iraq war at the time of its occurrence. Investigating the long-term effects of Iran-Iraq war over more than three decades after its end is also necessary. Therefore, considering the importance of the indirect effects of war on the mental health of the children of war veterans, the present study aimed to investigate the mediating the role of emotion regulation strategies in the relationship of metacognitive beliefs and attachment styles with risky behaviors among adolescent children of Iran-Iraq war veterans.

\section{Methods}

This is a descriptive-correlational study. The study population consisted of all male and female children of Iran-Iraq war veterans in Tehran, Iran, aged 12-17 years. Of these, 253 were selected and voluntarily responded to the short form of Cognitive Emption Regulation Questionnaire,
Metacognitions Questionnaire for Children, Risky Behavior Scale, and Collins and Reid's Revised Adult Attachment Scale. The collected data were analyzed using Structural Equation Modeling (SEM) in SPSS V. 18 and AMOS V. 22.

\section{Results}

According to goodness-of-fit indexes, the hypothesized model had good fit. Overall, the metacognitive beliefs, attachment styles and emotion regulation strategies explained $14 \%$ of the variance of risky behaviors. The results of path analysis showed that the direct effect of metacognitive beliefs on the adaptive emotion regulation strategies was not significant, but the direct effect of metacognitive beliefs on maladaptive emotion regulation strategies was significant. Furthermore, the direct effect of metacognitive beliefs on the risky behaviors of children was not significant. The direct effect of attachment styles on adaptive and maladaptive strategies and on the risky behaviors was significant. Moreover, the direct effect of adaptive and maladaptive emotional regulation strategies on the risky behaviors children was significant. Finally, the indirect effect of metacognitive beliefs and attachment styles on the risk behaviors was significant through the emotion regulation strategies (Figure 1).

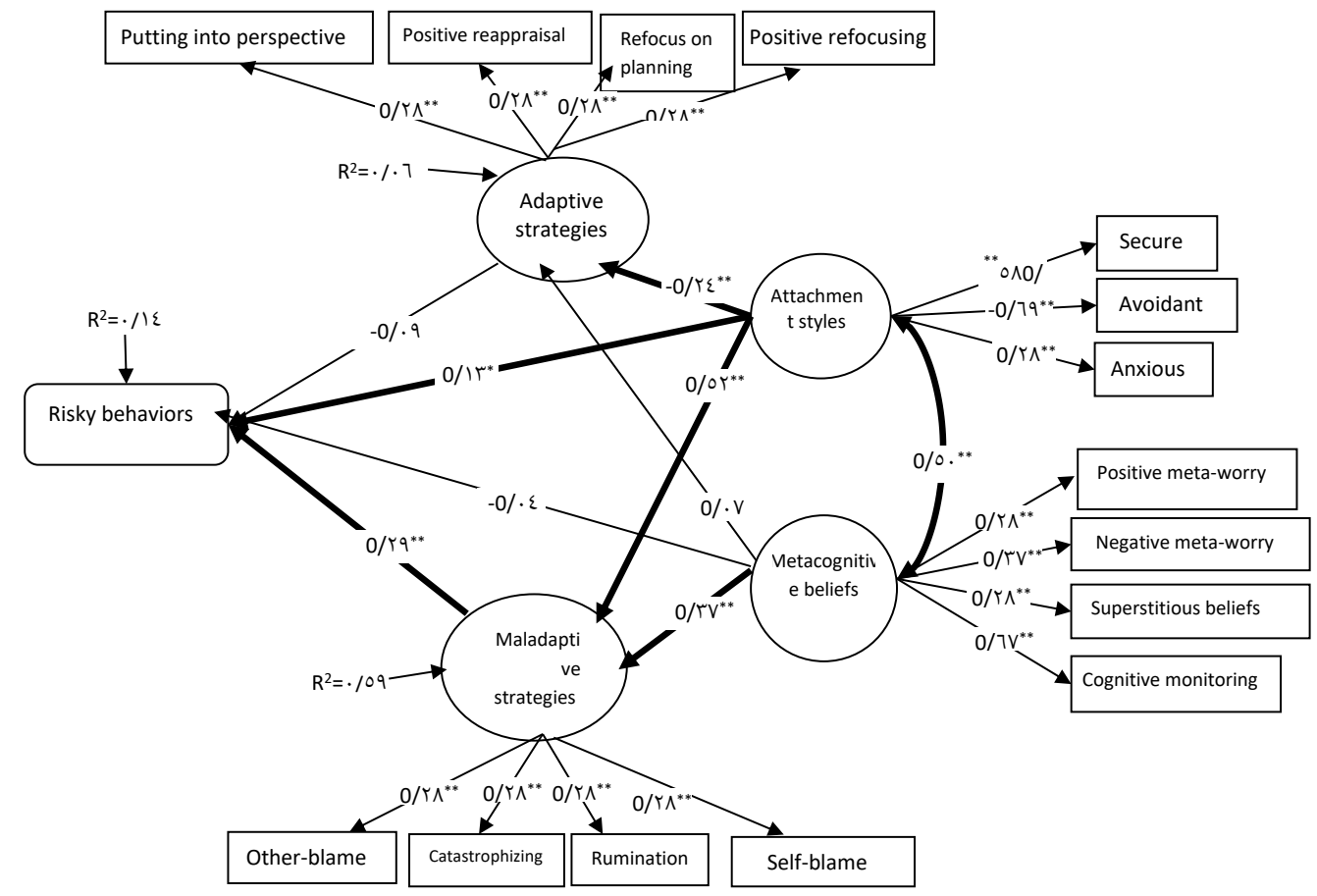

Iranian Journal of
PSYCHIATRY AND CUINICAL PSYCHOLOGY

Figure 1. Standard path coefficients, significance level, and coefficient of determination of study variables

Maximum likelihood estimation method; $* \mathrm{P}<0.05 ; * * \mathrm{P}<0.01$ 


\section{Discussion}

War as a severe stressful factor, has many social, economic, cultural and individual consequences affected the relationship of many generations of people [33]. The relationship between a war veteran suffering from psychiatric disorders and his/her children is often characterized by conflict, control, excessive proximity and excessive protection which cause the incidence of some psychological disorders among these children [36].

However, the fundamental mechanism of such effect is not clear yet. Given the importance of understanding the mechanism of psychological pathology in designing preventive and therapeutic interventions, the present study aimed to investigate the role of emotional regulation strategies(as a fundamental mediation process) in the relationship of metacognitive beliefs and attachment styles (as the underlying causes of psychopathology) $[8-10,12,13$, $17,37]$ with risky behaviors among adolescent children of war veteran suffering from psychiatric disorders. According to the results, it seems that adolescents in these families have various emotional problems, and some of them use strategies such as rumination, anxious attachment, avoidant attachment, etc. Using emotional regulation and metacognitive training interventions for effective management of inevitable tensions and stresses associated with living with a war veteran can be very helpful in preventing risky behaviors in their children [42]. Metacognitive therapy may also help reduce the emotional problems and risky behaviors such as substance abuse [43, 44].

\section{Ethical Considerations}

\section{Compliance with ethical guidelines}

An informed consent was obtained from the participantsafter explaining the research objectives and method. Theywere also free to leave the study at any time and were assured of the confidentiality of their information.

Funding

This research received no specific grant from fundingagencies in the public, commercial, or not-for-profit sectors.

\section{Authors contributions}

Conceptualization, methodology, initial draft preparation: All authors; Investigation, supervision and project administration: Nasibeh Yazdan Panahi and Shahram Mohammad- khani; Resources, editing and review: Shahram Mohammadkhani, Jafar Hasani and Mehdi Akbari.

\section{Conflicts of interest}

The authors declared no conflicts of interest.

\section{Acknowledgements}

The authors would like to thank all the participants for their cooperation in this research. 


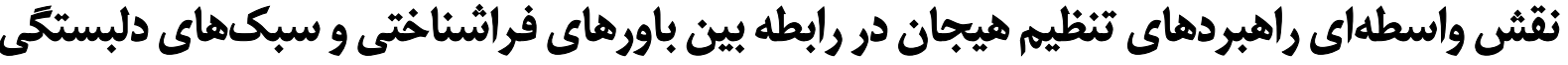

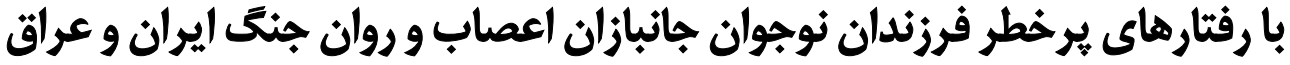

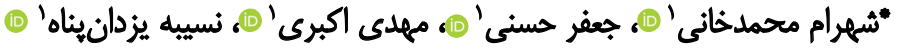 \\ ا. كروه روانشناسى بالينى، دانشكده روانشناسى و علوم تربيتى، دانشعاه خوارزمى، تهران، ايران.
}

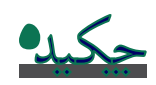

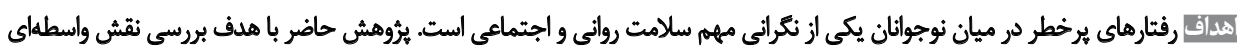

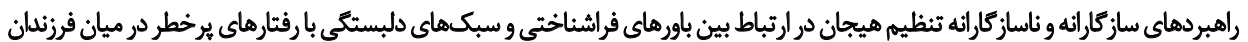

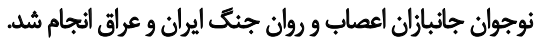

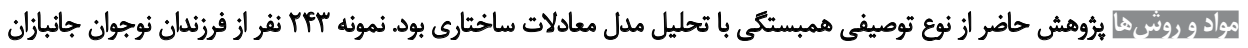

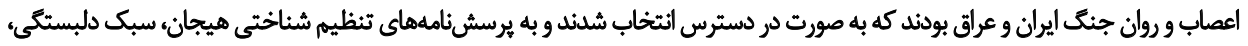

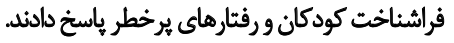

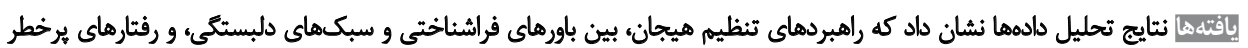

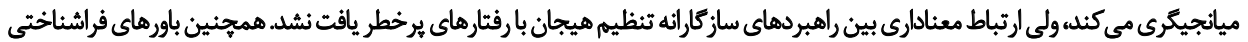

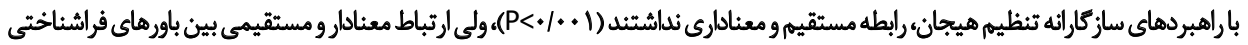

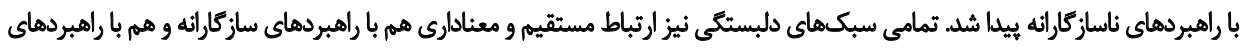

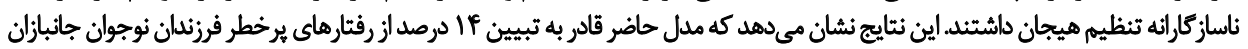
اعصاب و روائ است.

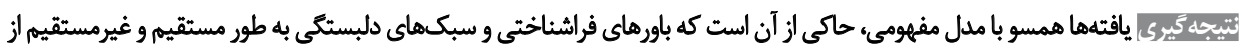

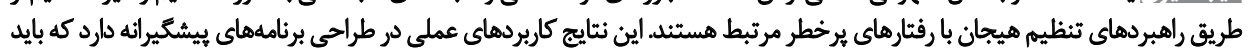
مورد توجه قرار كيرد
\end{abstract}

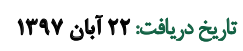

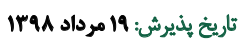

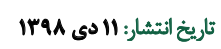

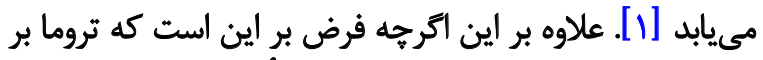

dale.

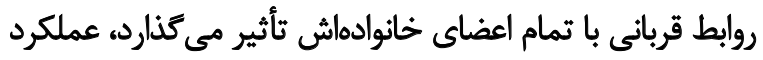

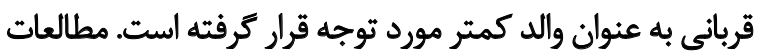

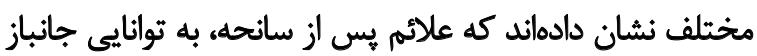

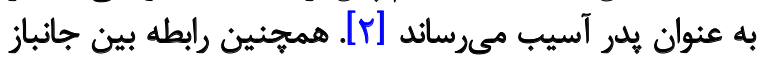

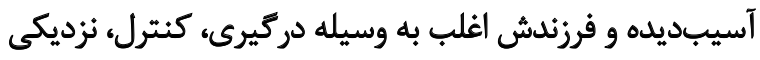

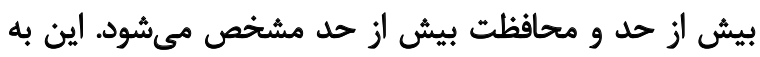

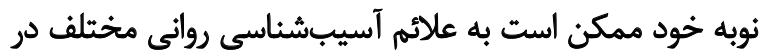

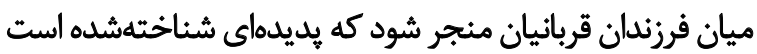

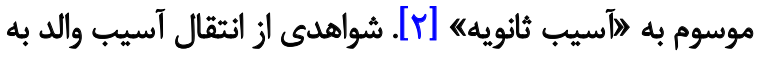

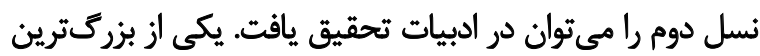

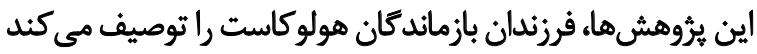

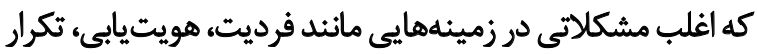

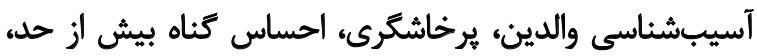

\section{كليدوازهها: - تئها:

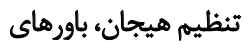

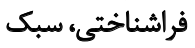

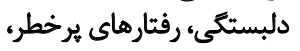

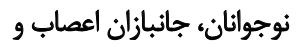 روان جنى ايران و عراق أناب}

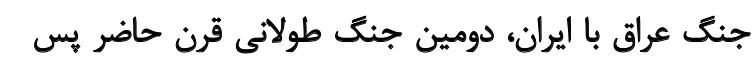

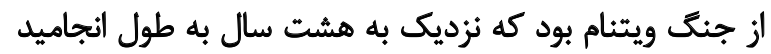

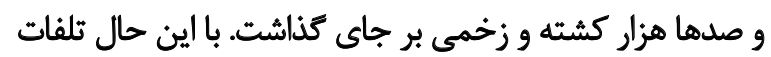

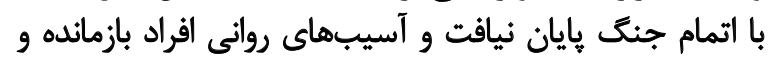

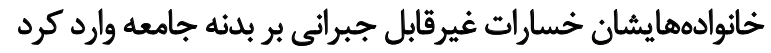
و اثرات نامطلوب آن همجنان آنانئ ادامه دارد.

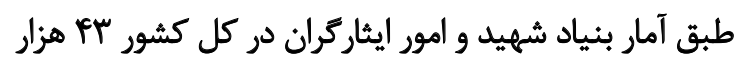

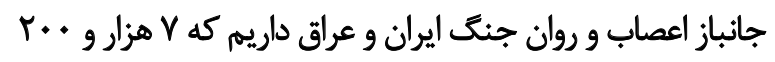

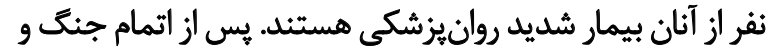

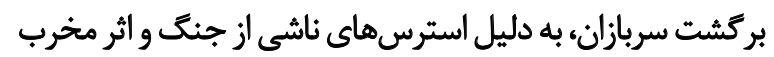

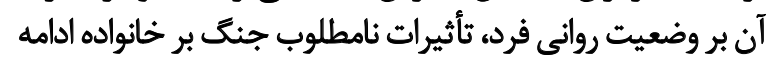

$$
\text { - }
$$

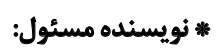

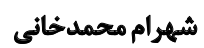
نشانى: تهران، دانشعاه خوارزمى، داتشكده روانشناسى و علوم تربيتى كروه روانشناسى بالينى. تلفن: بست الكترونيكى:mohammadkhani@khu.ac..ir 
نشاندهنده نقص در مهارتهاى مقابله (مانند تنظيم هيجان

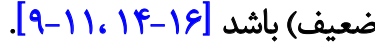

عامل ديكرى كه در بروز رفتارهاى يرخطر مؤثر است، سبكهاى

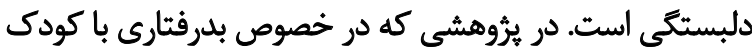

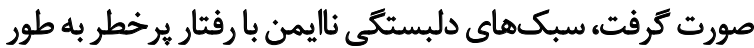

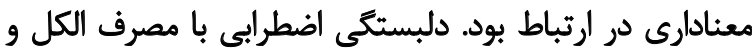

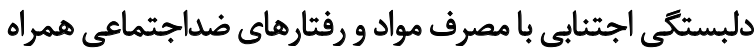

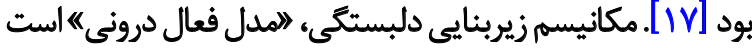

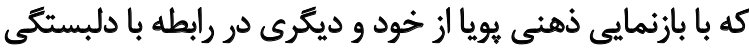

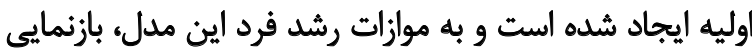

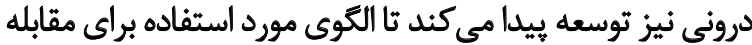

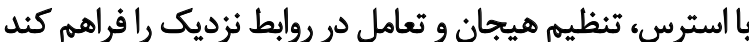

افراد ايمن، مدل كار درونى مثبتى هم از خود و هم از از ديكران

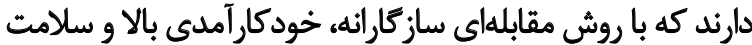

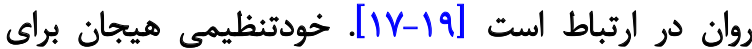

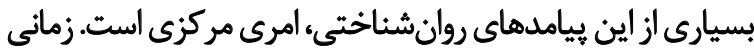

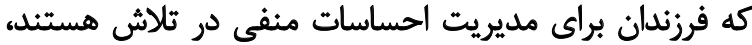

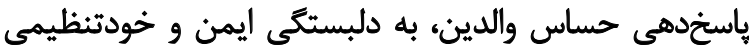

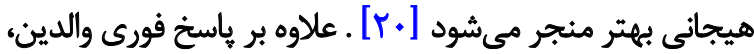

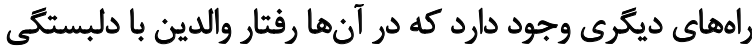

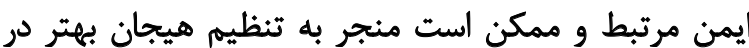

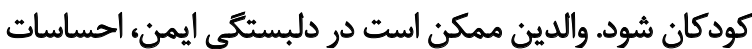

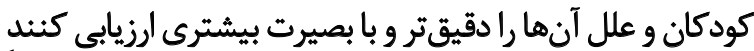

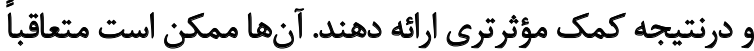

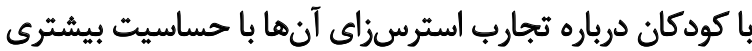

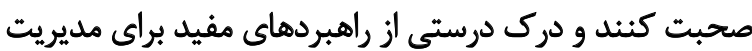
اين احساسات ارائه دهند.

در كوتامدت، ويرگى هاى مرتبط با دلبستَى ايمن، ممكن

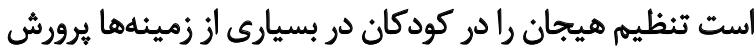

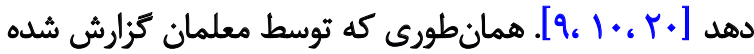

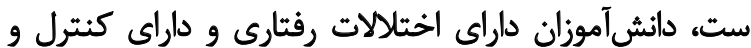

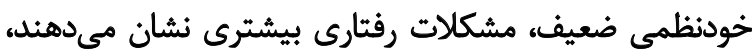

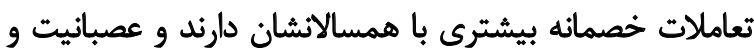
يرخاشكرى از خود نشان مي دهند [9].

در كشور ما كه يكى از قربانيان جنك است، اكثر تحقيقات

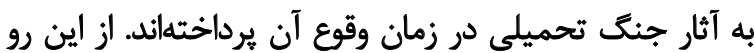

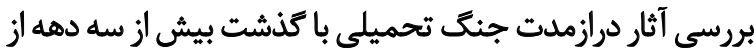

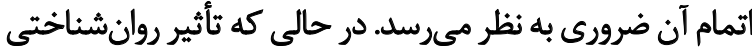

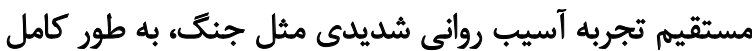

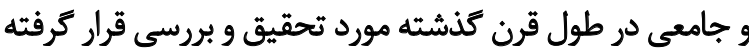

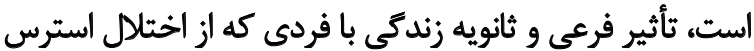

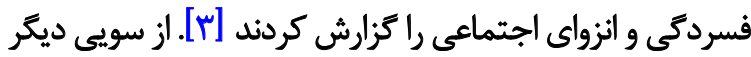

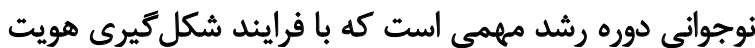

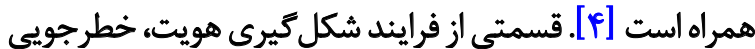

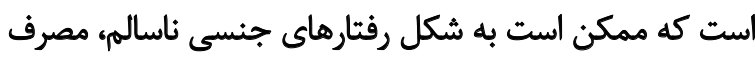

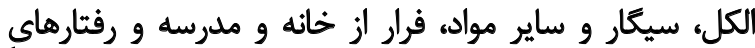

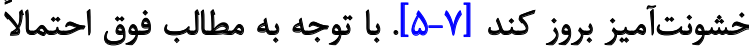

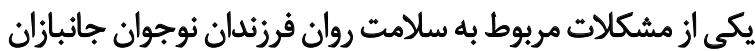

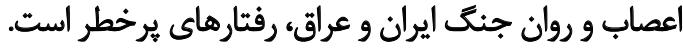

در طول دهه كذشته، محققان به طور فزايندهاى به شناسايى

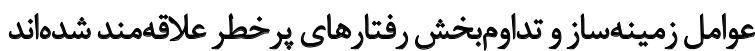

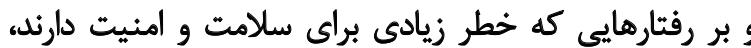

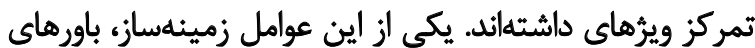

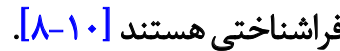

مطابق با نظريه فراشناختى اختلالات روانشناختى [11]؛

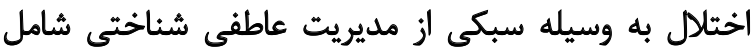

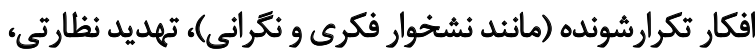

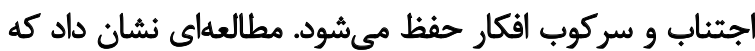

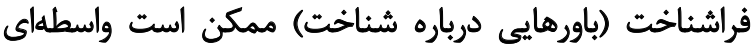

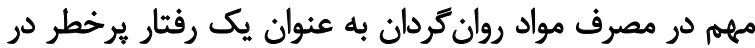

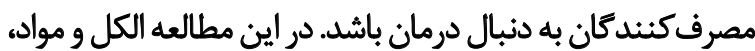

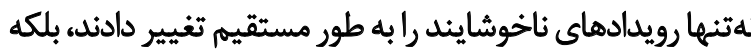

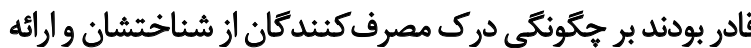
آنها با شدت و نار احتى كمتر درك، اثر كذارند.

باورهاي فراشناختى ممكن است يك مانع بالقوه براى درمان

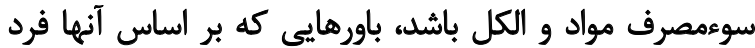

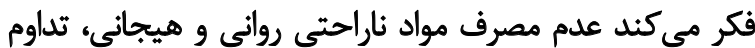

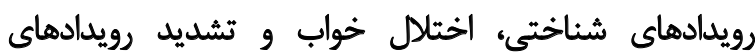

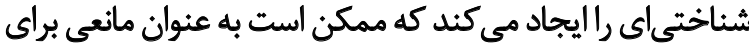

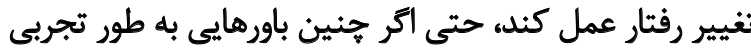

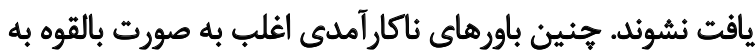

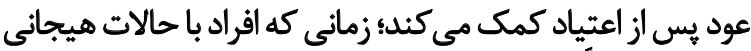

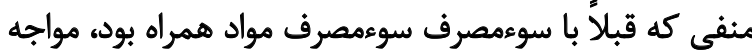

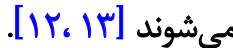

بر اساس اين مدل، باورهاى فراشناختى مثبت شامل باورهايى

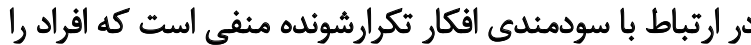

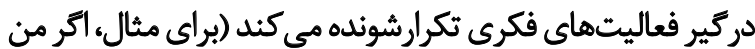

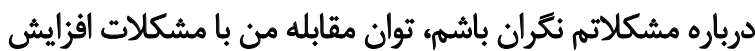

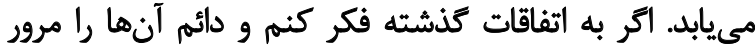

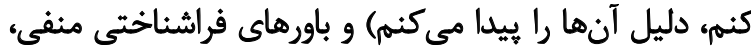

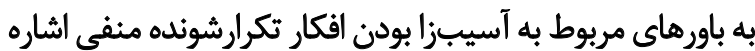

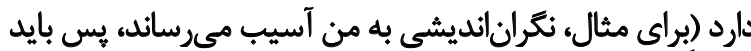

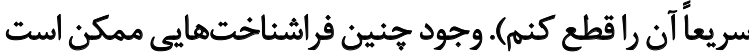




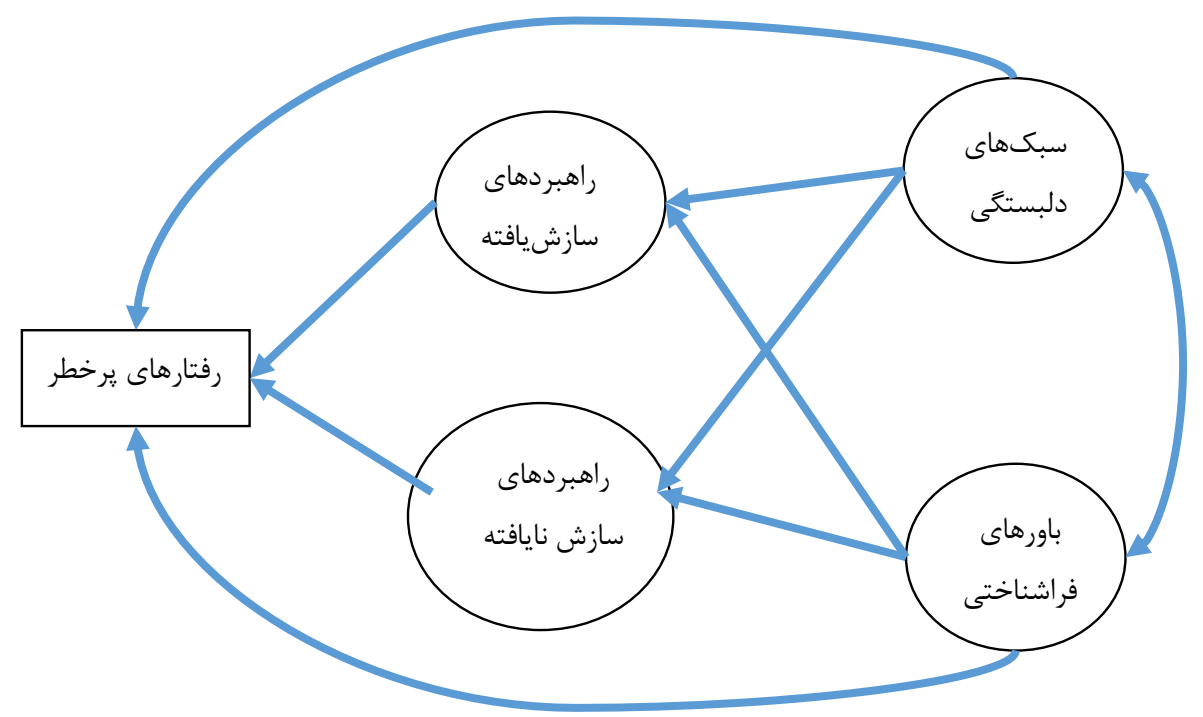

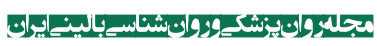

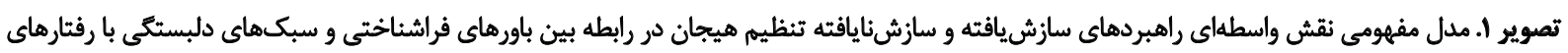

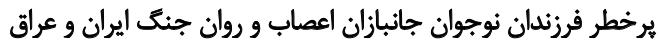

شد و بر اساس اطلاعات يرونده تحصيلى و هماهنكى مديران

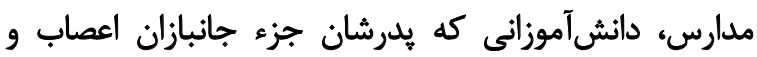

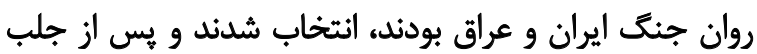

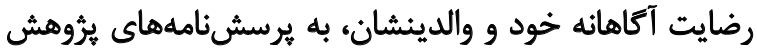

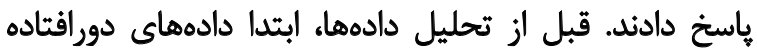

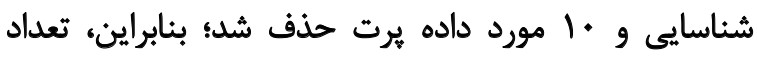

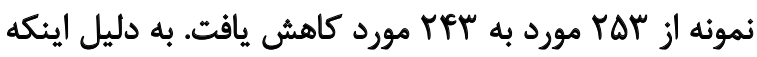

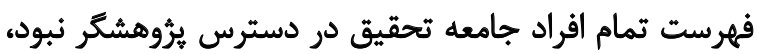

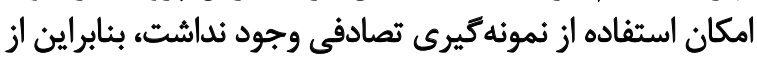

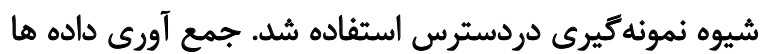

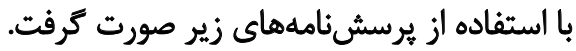

\section{فرم كوثاه يرسش نامه تنظيم شئاختى هيبان'}

اين يرسشنامه توسط كارنفسكى و كرايج و اسيينهاون [YI)

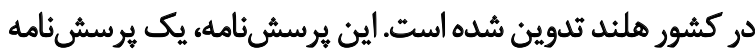

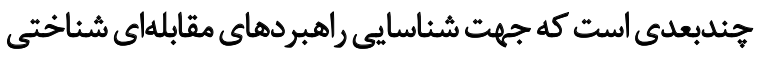

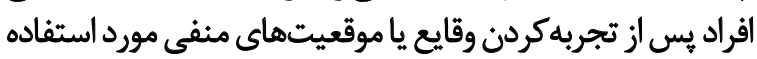

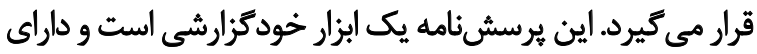

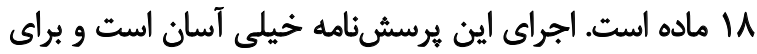

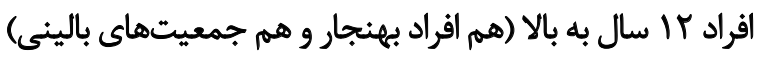

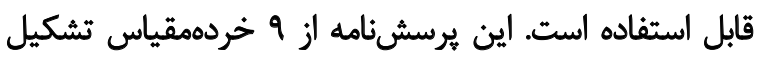

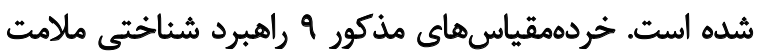

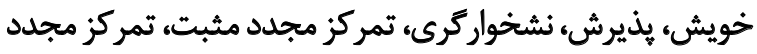

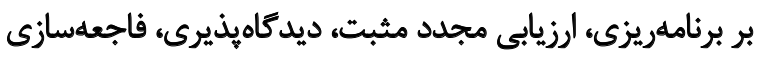

1. Cognitive Emotion Regulation Questionnaire (CERQ-SHORT)
يس از سانحه رنج مىبرد به ميزان بسيار كمترى مورد تحقيق و بررسى قرار كرفته است.

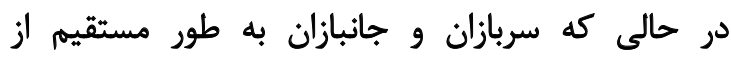

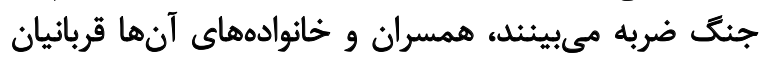

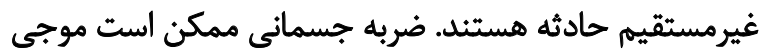

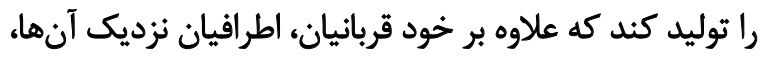

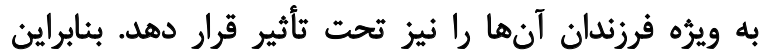

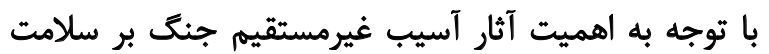

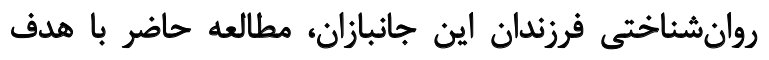

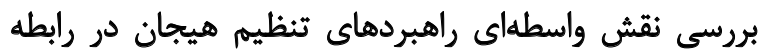

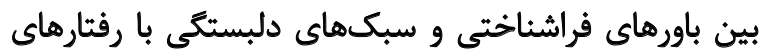

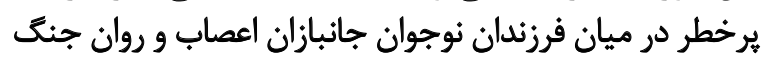

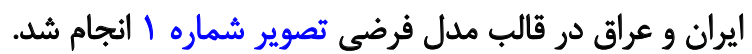

يروهش حاضر از نوع توصيفى همبستكى با تحليل مدل

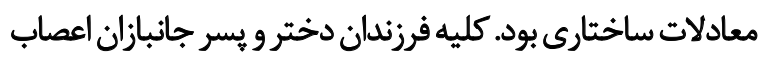

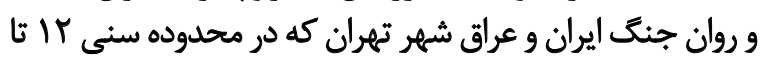

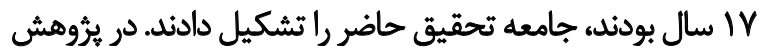

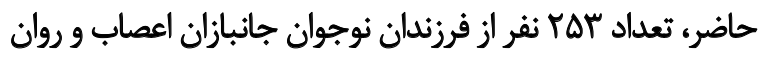

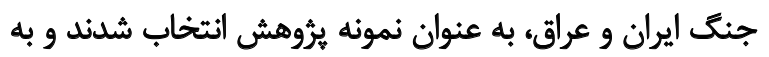

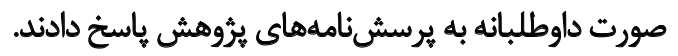

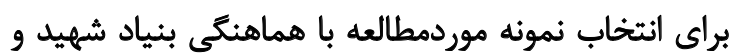

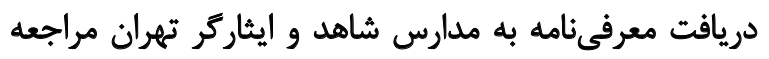




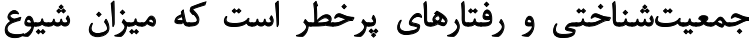

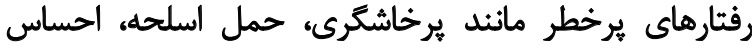

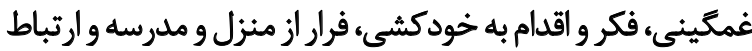

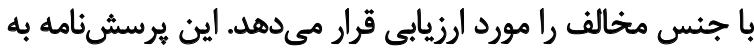

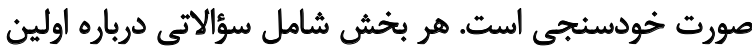

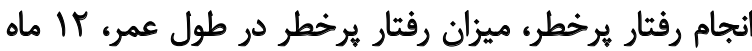

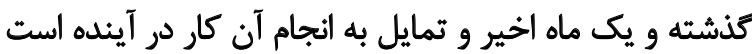

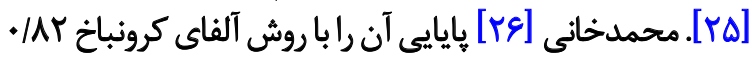

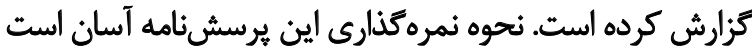

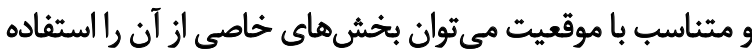

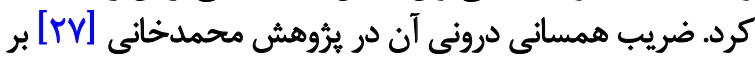

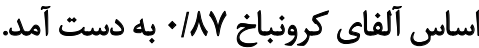

\section{مقياس سبك دلبسيثكي بكوليثز و ويد}

اين مقياس شامل خودارزيابى از مهارتهاى ايجاد روابط

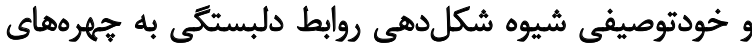

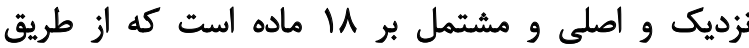

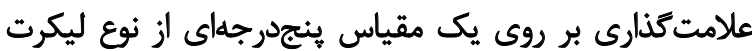

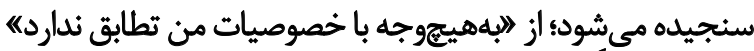

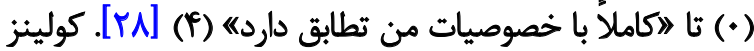

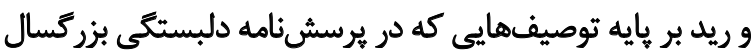

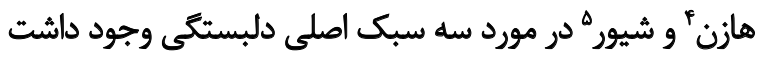

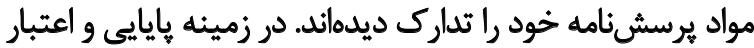

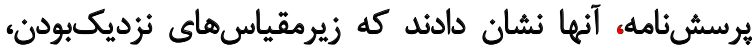

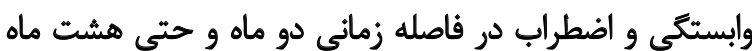

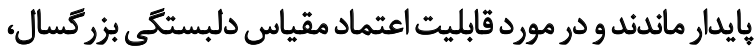

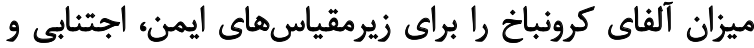

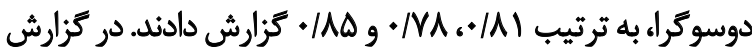

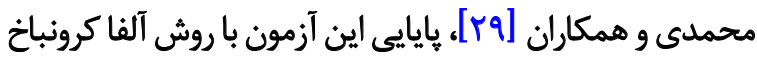

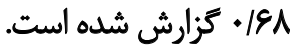

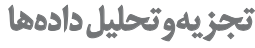

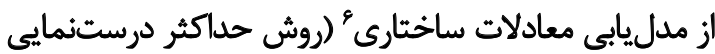

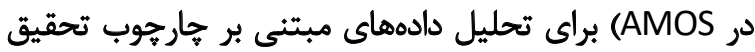

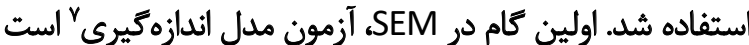

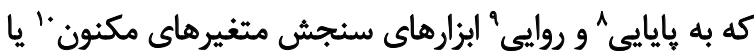

3. Revised Adult Attachment Scale

4. Hazan

5. Shaver

6. Structural Equation Modeling (SEM)

7. Measurement model

8. Reliability

9. Validity

10. Latent variable
و ملامت ديكران را ارزيابي مى كنيند. دامنه نمرات مقياس، از 1 إن

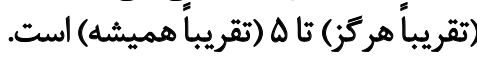

نمرات بالا در هر خردهمقياس بيانكّر ميزان استفاده بيشتر

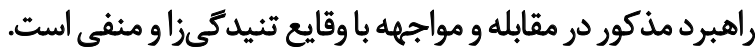

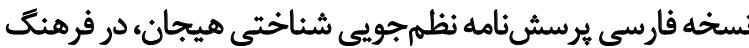

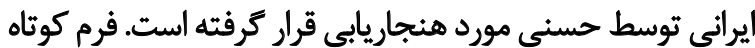

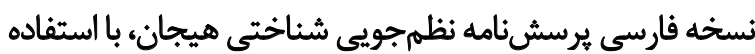

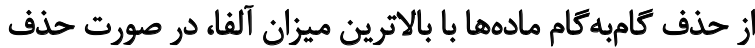

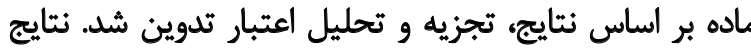

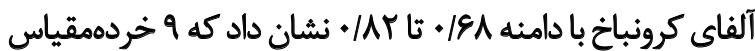

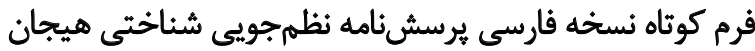

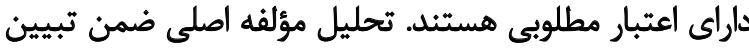

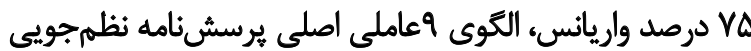

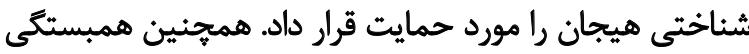

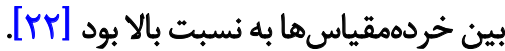

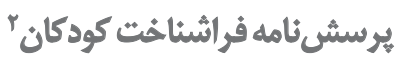

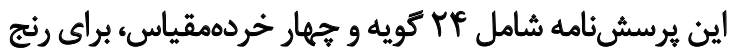

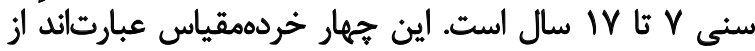

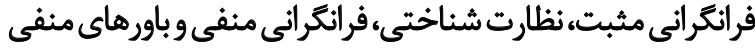

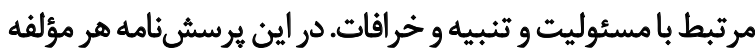

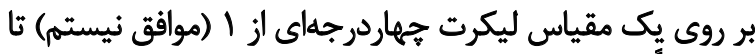

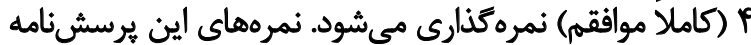
از ئ Ff Ff

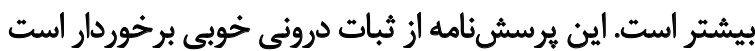
(A)=/AV)

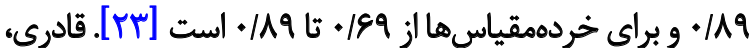

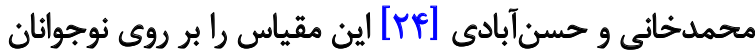

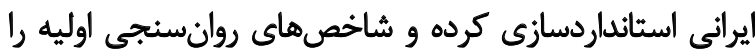

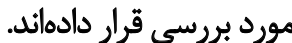

ساختار عاملى اين مقياس با استفاده از روش تحليل عاميلى

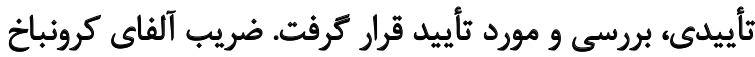

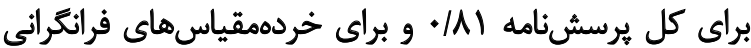

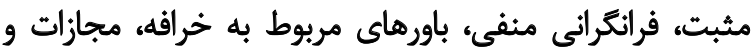

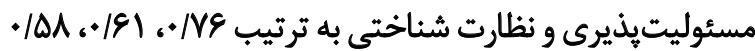

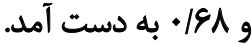

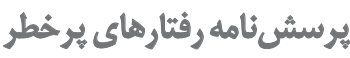

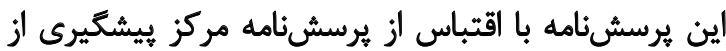

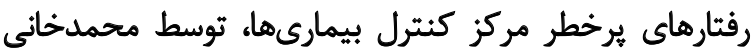

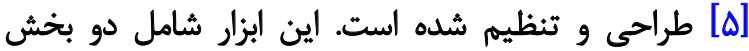

2. Metacognitions Questionnaire for Children (MCQ-C) 
در دامثه قابل قبول تا مطلوب قرار كرفتند. يُّ از آن آمارههاي

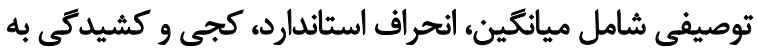

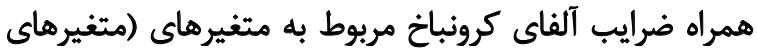
مشاهدهده) يُروهش مورد بررسى قرار ترفت.

نتايج جدول شماره ا نشان ميدهد كه ضرايب يايايى (آلفاى

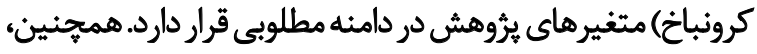

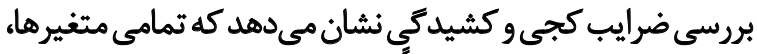

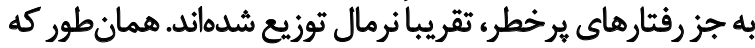

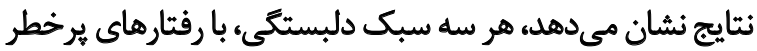

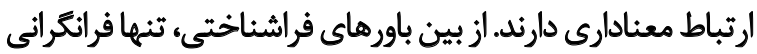

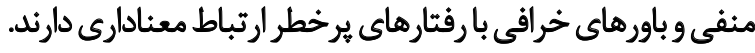

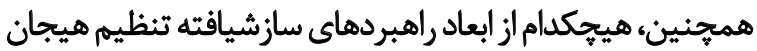

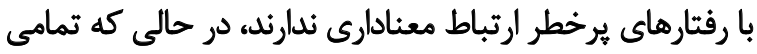

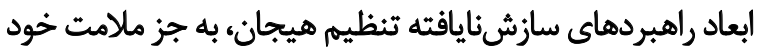

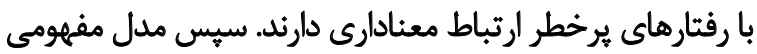

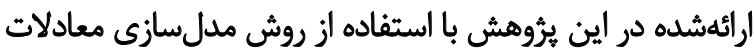

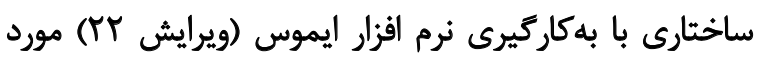

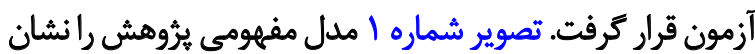

مي دهد.

براى تحليل اين مدل، با توجه به مدل مفهومى يُروهش،

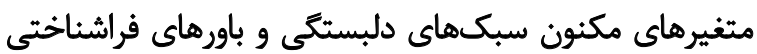

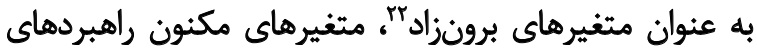

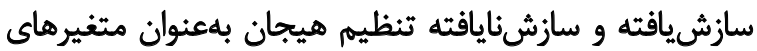

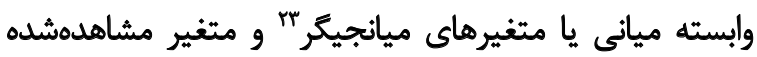

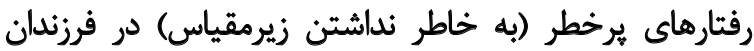

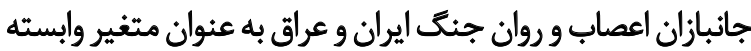

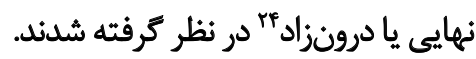

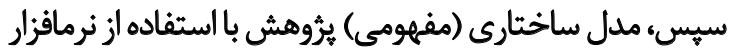

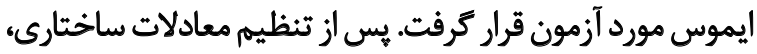

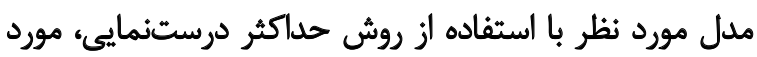

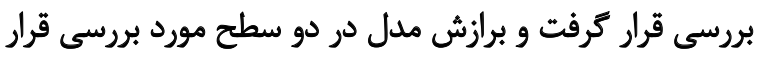

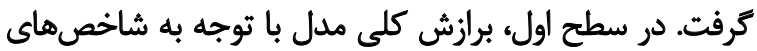

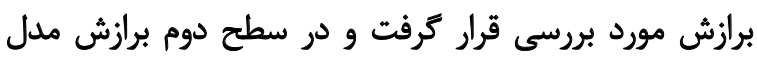

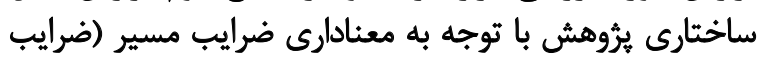

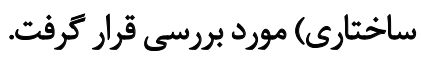

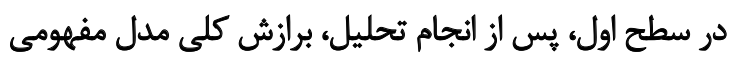

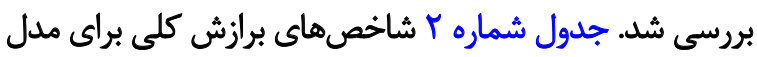

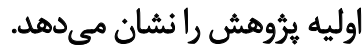

همانطور كه نتايج جدول شماره ا نشان مى دهد شاخص خى ليى

22. Exogenous

23. Mediator

24. Endogenous
به عبارت بهتر به روابط متغيرهاى مشاهده شده" با متغيرهاي

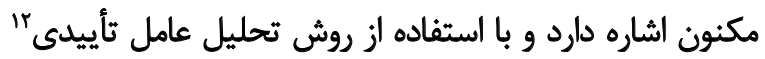

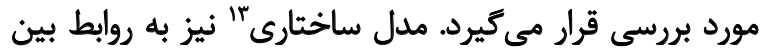

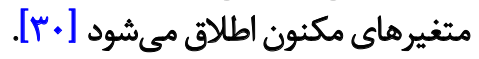

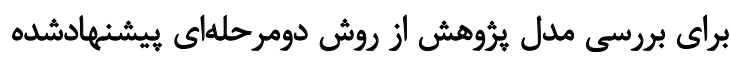

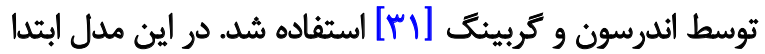

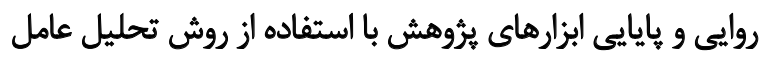

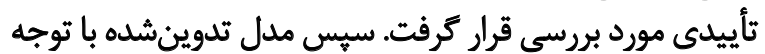

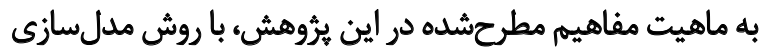

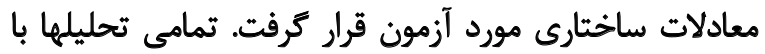

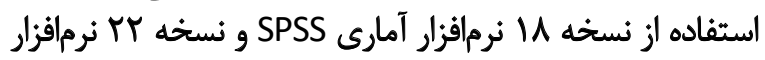

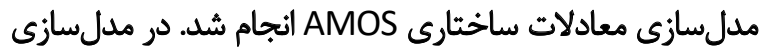

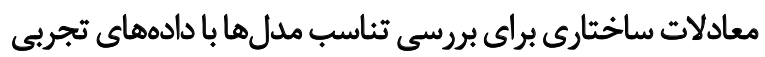

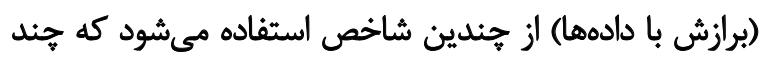

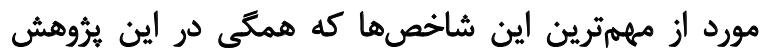

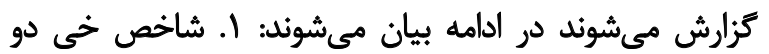

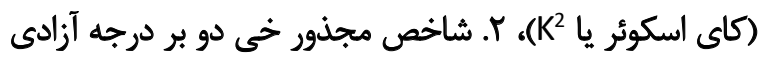

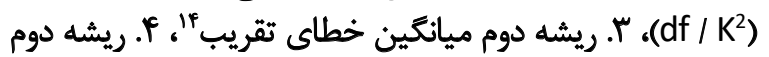

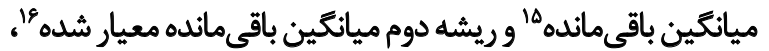

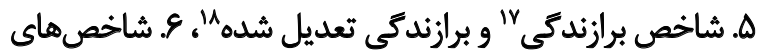

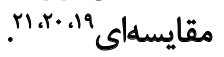

1.

براى بررسى مدلهاي اندازهيرى يُووهش بر اساس مدل

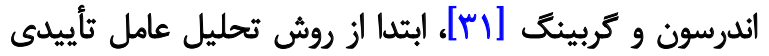

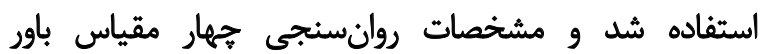

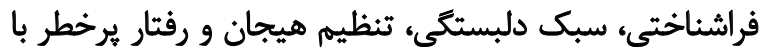

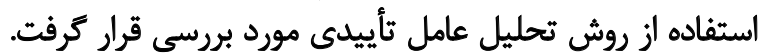

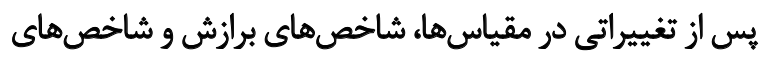

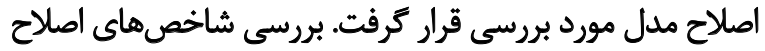

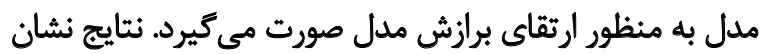

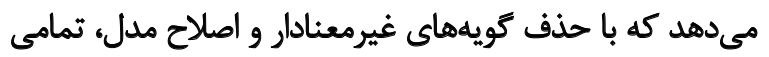

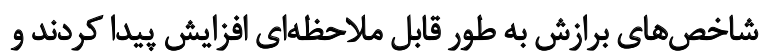

11. Observed variables

12. Confirmatory factor analysis

13. Structural model

14. Root Mean Square Error of Approximation (RMSEA)

15. Standardized SRMR

16. Standardized RMR

17. Goodness of Fit Index (GFI)

18. Adjusted Goodness of Fit Index (AGFI)

19. Normed Fit Index (NFI)

20. Non-Normed Fit Index (NNFI)

21. Comparative Fit Index (CFI) 
جدول ا. آمارههاى توصيفى مربوط به متغيرهاى يُروهش

\begin{tabular}{|c|c|c|c|c|}
\hline ضريب آلفا & ميانكين $\mid$ ميحراف|ستاندارد & تعداد كويهها & 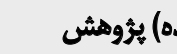 & متغيرهاى \\
\hline$\cdot / M^{2}$ & $1+/ \Delta \Delta \pm r / \Delta r$ & $r$ & ايمن - - ايمن & \multirow{3}{*}{ سبكهاى دلبستكى } \\
\hline . IFA & $1 . / r \lambda \pm r / q 1$ & $\Delta$ & إجتنابى & \\
\hline.$/ N S$ & $11 / 4+ \pm 8 / .8$ & 8 & اضطرابي & \\
\hline.$|A|$ & $\mid r / N \pm \pm / R g$ & 8 & فرانكرائي مثبت & \multirow{4}{*}{ باورهاي فراشناختي } \\
\hline.$/ N^{\infty}$ & $1 \% / 99 \pm r / V A$ & $\Delta$ & فرائكرائى منفى & \\
\hline$\cdot / v$ & $11 / p r \pm r / p q$ & $\Delta$ & باورهاى خرافي & \\
\hline - $/ A r$ & $\mid V / \cdot V \pm P / \pi r$ & 8 & نظظارت شناختى & \\
\hline$\cdot / \Delta V$ & $\Delta / r T \pm r / M r$ & r & تمركز مثبت & \multirow{5}{*}{ راهبردهاي سازش شيافته } \\
\hline .180 & $e / p q \pm r / 19$ & r & تمركز ارزيابى & \\
\hline$\cdot 101$ & $g / f) \pm r / M$ & r & الرزيابى مجدد & \\
\hline $.1 \Delta 8$ & $\Delta / q Y \pm r / \cdot \varepsilon$ & r & ديدكاهكيرى & \\
\hline$+|A|$ & $r T / q Y \pm \Delta / q^{\mu}$ & $\Lambda$ & 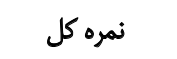 & \\
\hline$\cdot M^{e}$ & $r / q q \pm r / \cdot q$ & r & ملامت خود & \multirow{5}{*}{ راهبردهاى سازث ناياقته } \\
\hline $.19 V$ & $\Delta / a r \pm r / A V$ & r & نشخواركرى & \\
\hline .18 & $r / \Lambda a \pm r / \cdot V$ & r & فاجعهسازى & \\
\hline.$/ A Y$ & $P M E \pm T / M r$ & $r$ & ملامت ديكران & \\
\hline . $\mathrm{NA}$ & $r \cdot / \Delta \odot \pm \Delta / ৭ q$ & $\wedge$ & نمرهكل & \\
\hline . $/ A r^{2}$ & r/Ratr/gre & m & \multicolumn{2}{|c|}{ رفتارهاى برخطر } \\
\hline
\end{tabular}

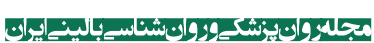

با بين حال، تمامى شاخصهاي برازش كلى براي مدل برُوهش

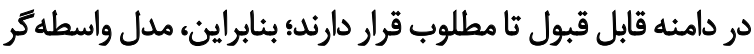

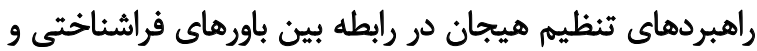

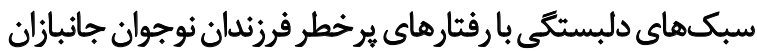

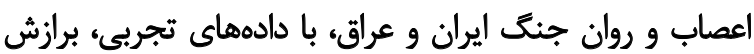

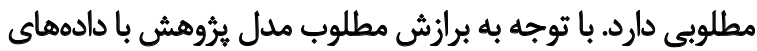

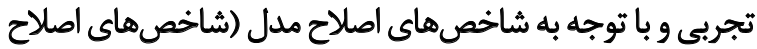

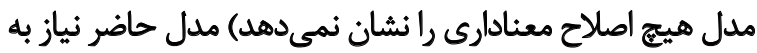

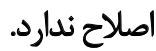
در سطح دوم، يس از تحليل اوليه، ضرايب مسير مدل و ضرايب تعيين متغيرهاى درونزا مورد بررسى قرار كرفت. تصوير شماره
دو معنادار است. شاخص خحى دو، برازش مدل را به طوري موركلى مورد

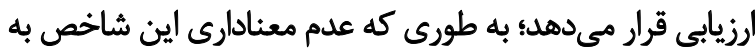

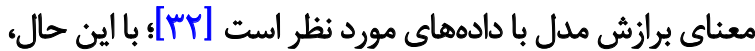

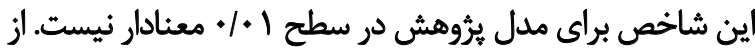

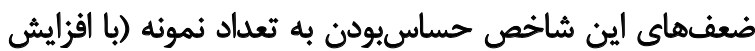

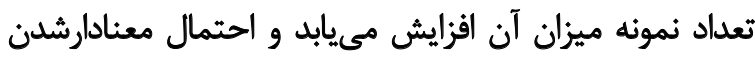

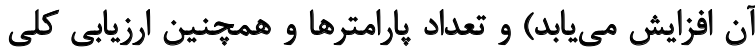

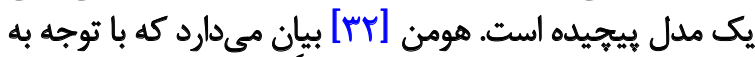

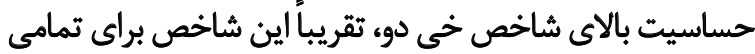

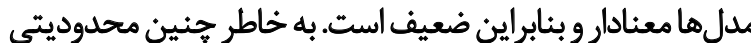
بايد به شاخصهاى ديكر رجوع كرد.

جدول r. شاخصهاى برازش كلى مربوط به مدل يرُوهش

\begin{tabular}{|c|c|c|c|c|c|c|c|c|c|c|c|}
\hline SRMR & RMSEA & CFI & TLI & NFI & AGFI & GFI & $K^{2} / d f$ & df & $\mathbf{P}$ & $K^{2}$ & شاخصشهاي \\
\hline \multicolumn{2}{|c|}{ هايينتو از || } & \multicolumn{5}{|c|}{ بالاتو از A| - } & \multicolumn{2}{|c|}{ كمتر ازي } & \multicolumn{2}{|c|}{ معنادارى آزمون } & دامنه مورد قيول \\
\hline \multirow[t]{2}{*}{$.1 .8 V$} & .1 .89 &.$/ 91$ & .119 & . /NA & $\cdot / M$ & $+/ 91$ & r/IV & $\vartheta$ & $\%$ & $r+V / V$ & مدل بثروهش \\
\hline & مطلوب & & & ل ل ل قيول & & & مطلوب & & & & وضعيت \\
\hline
\end{tabular}




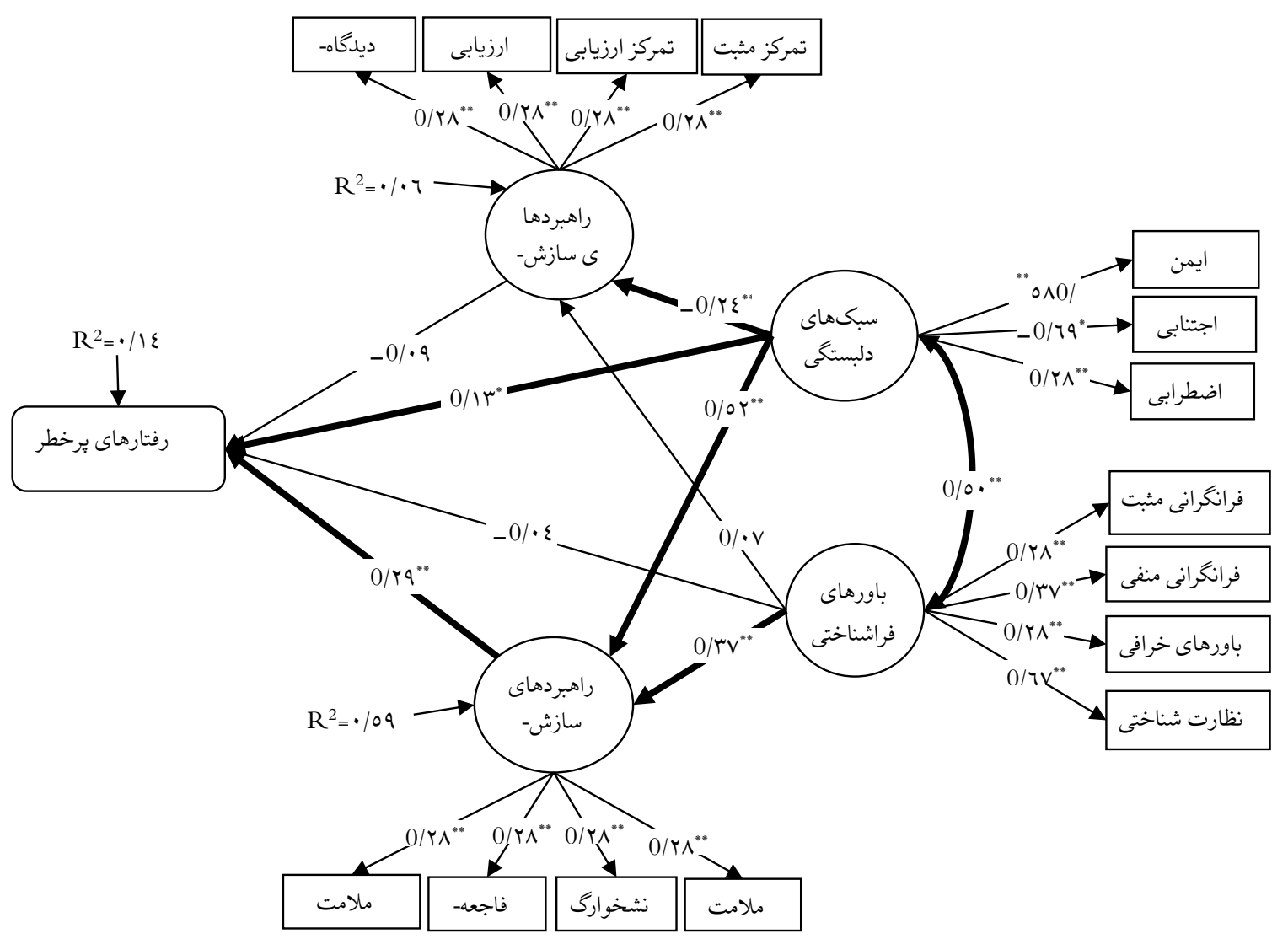

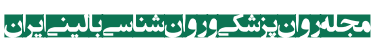

تصوير r. ضرايب مسير استانداردشده، معنادارى و ضرايب تعيين متغيرهاى درونزاي مدل

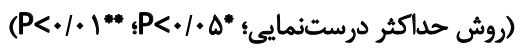

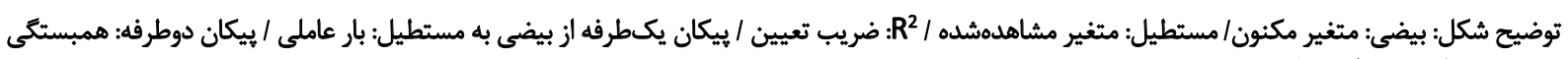
بين متغير هاي برونزاي مدل بيفي

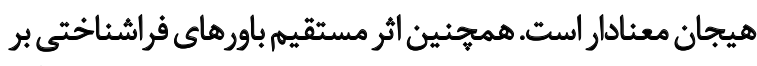

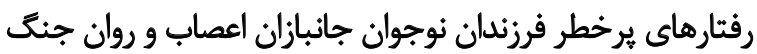

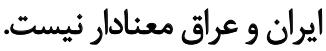

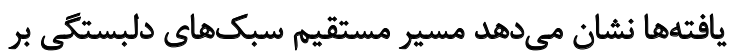

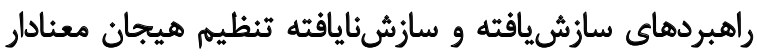

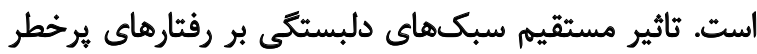

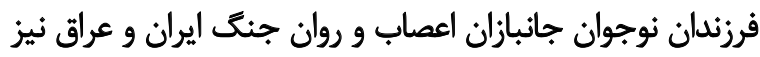

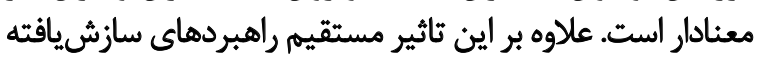

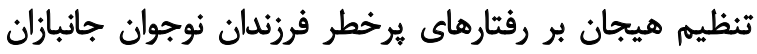

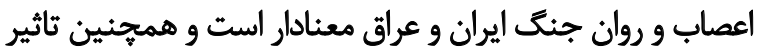

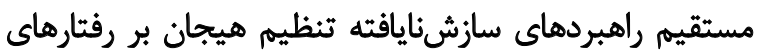

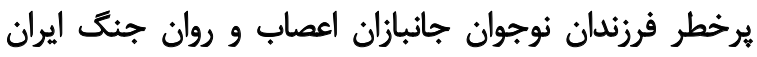

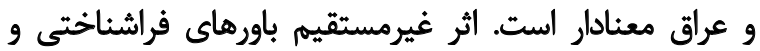

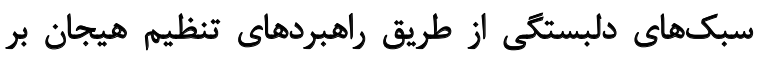

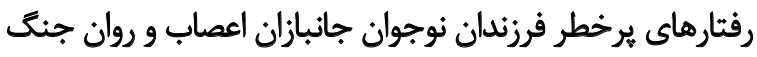

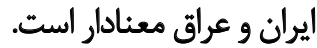

ז اين نتايج را نشان مىدهد (مسيرهاى معنادار يررنتتر نمايش داده شده است).

نتايج ارائهشه در تصوير شماره Y نشان ميدهد كه من تنها

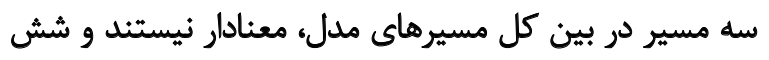

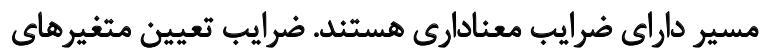

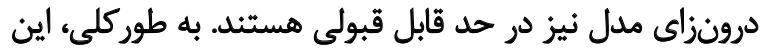

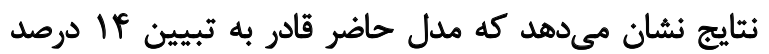

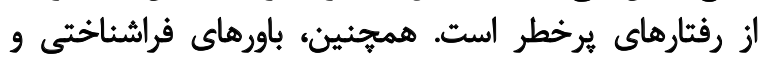

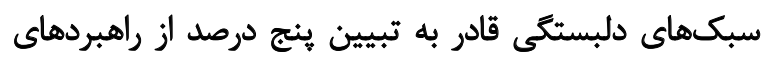

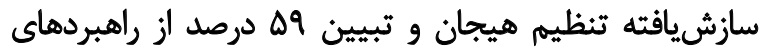

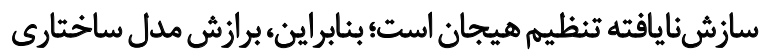
ئروهش در حد مطلوب است.

نتايج يُووهش نشان مى دهد كه اثر مستقيه باورهاى فراشناختى

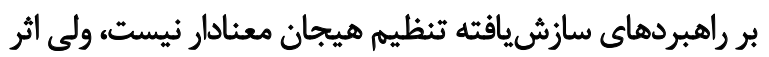

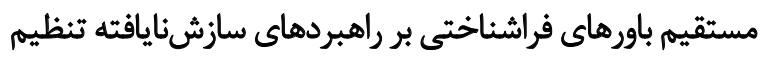


خشهم، مشكلاتي در ايجاد و حفظ دلبستكى و بسيارى اختلالات

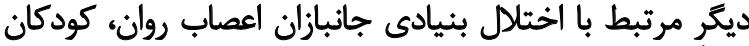

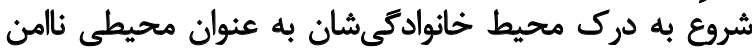

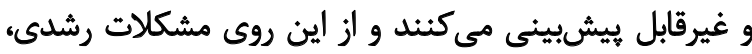

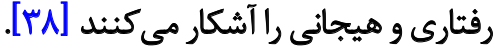

نهتنها تمام كودكان دلبستَى ايمن با والدينشان ندارند، بلكه

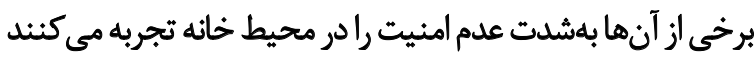

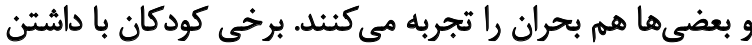

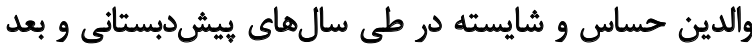

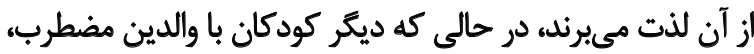

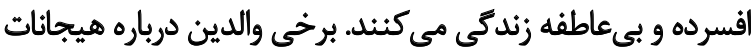

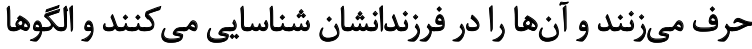

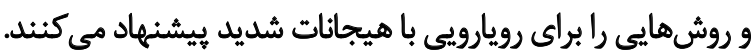

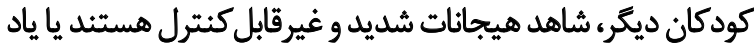

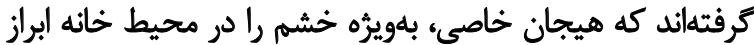

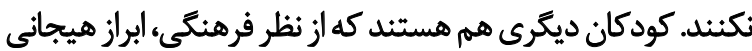

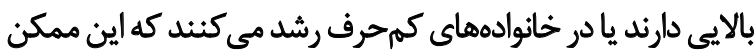

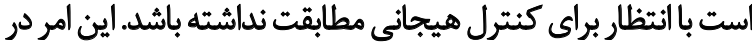

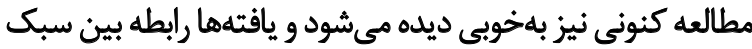

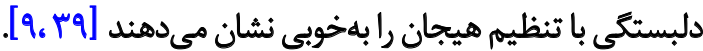

مدل فعال سازي و يويايى هاى سيستم دلبستكى توسط شيور

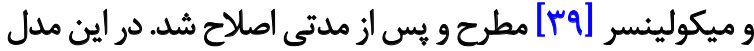

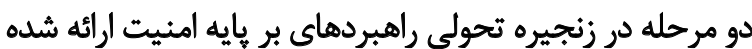

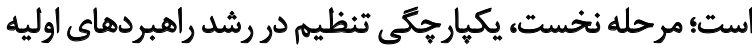

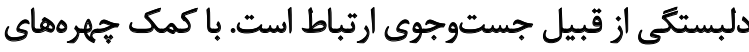

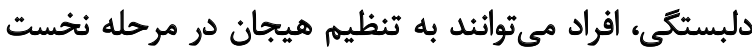

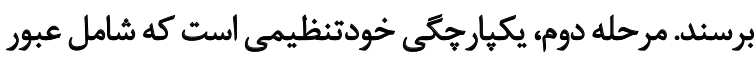

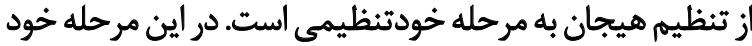
به عنوان نماينده اجراى راهبردهاى بر هايه امئيت، عمل مي كئند. فرزندان جانبازان اعصاب و روان شايد به دليل عدم حضور

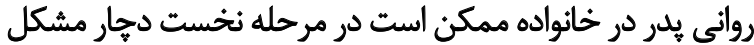

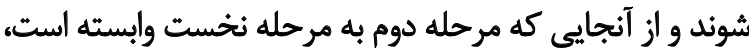

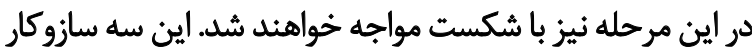

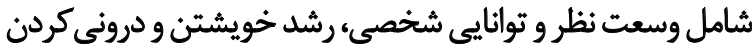

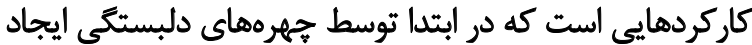

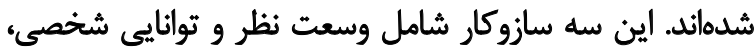

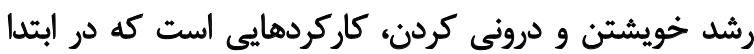

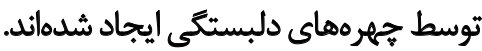

تنظيم هيجان مهارتى بنيادي محسوب مي رشود كه عملكرد

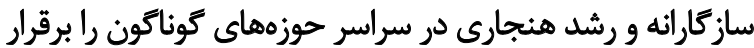

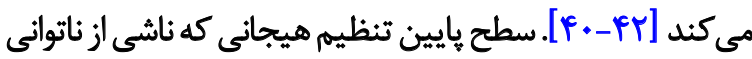

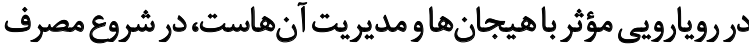

بحث

جنَّ به عنوان يك عامل فشارزاي شديد، بيامدهاي اجتماعى،

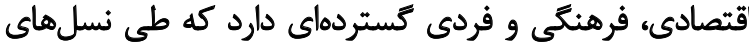

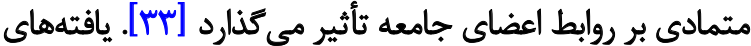

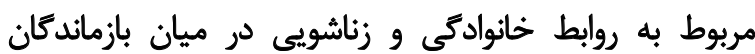

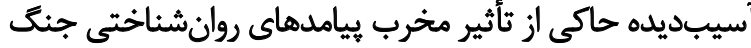

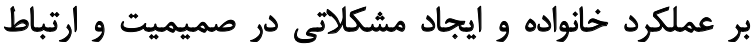

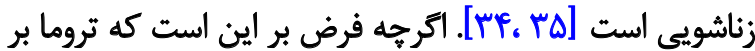

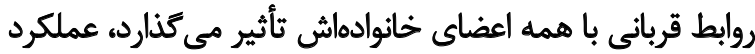

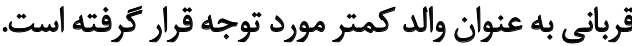

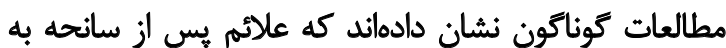

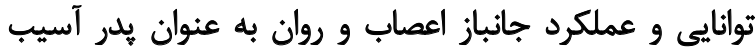

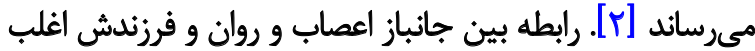

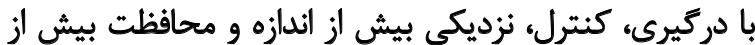

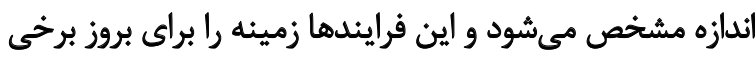

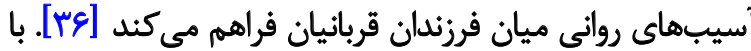

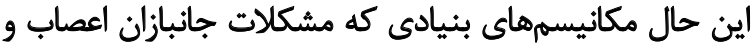

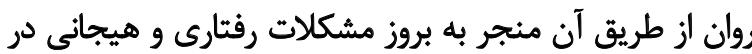

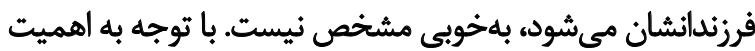

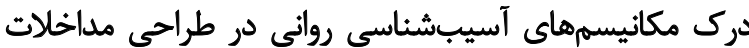

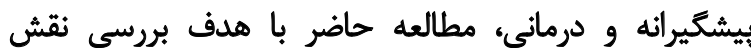

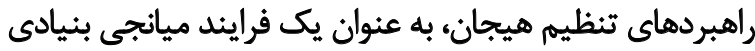

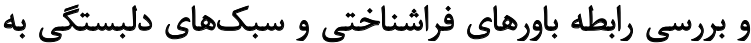

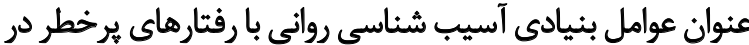

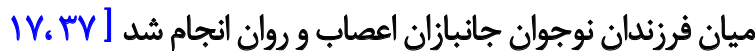

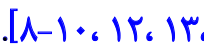
همسو با مطالعات ديكر، يافتههاى يُروهش نشان داد كه

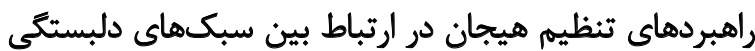

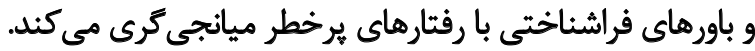

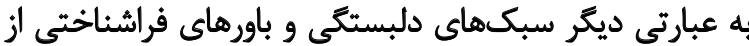

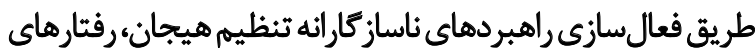

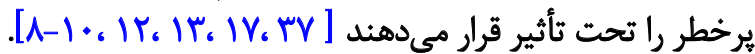

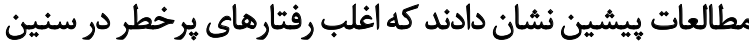

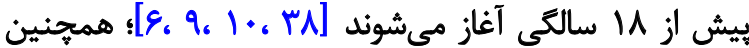

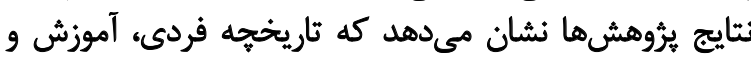

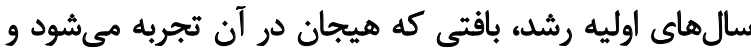

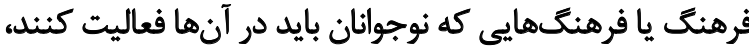

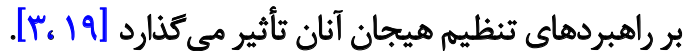

با توجه به اندوه حلنشده جانبازان اعصاب و روان و ترسي از ازئ

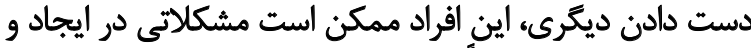

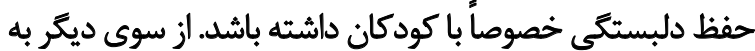
سبب انسجام كم خانواده و عدم دسترسي هيجاني انيى جانباز، طغيان 


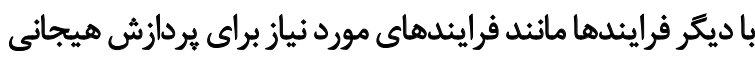

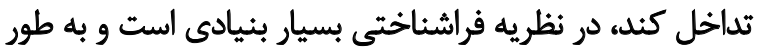
مستقيم مورد ارزيابى قرار كرفته است.

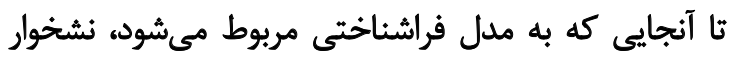

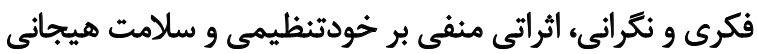

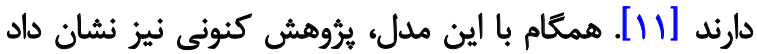

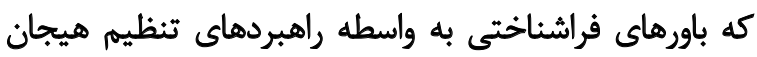

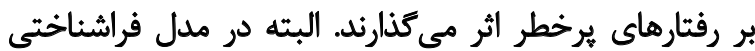

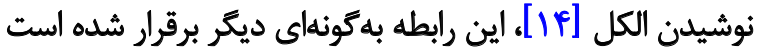

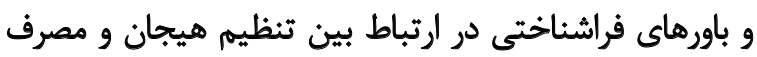

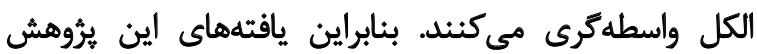

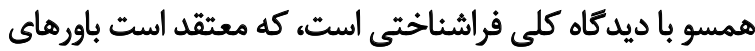

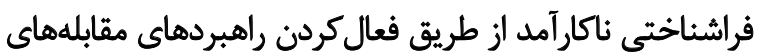

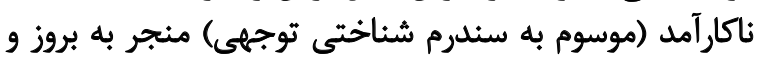

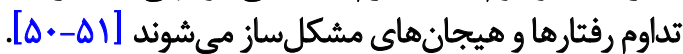
از ديرباز مشخص شده است كه اختلالات روانى دروالد مى تونواند

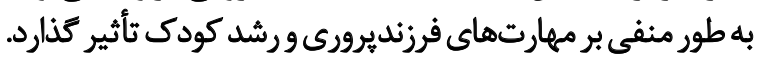

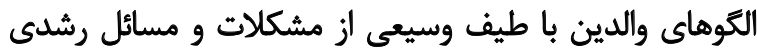

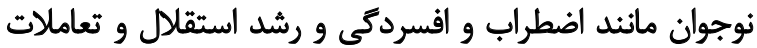

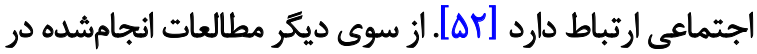

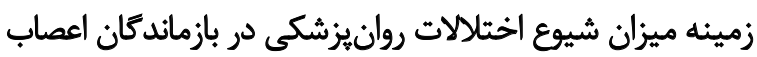

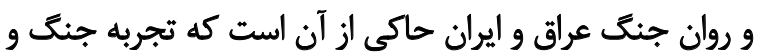

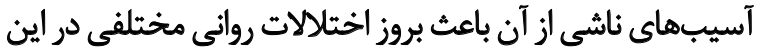

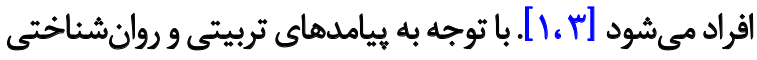

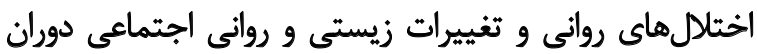

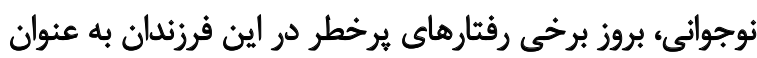
نوعى راهبرد مقابلهاى ناكار آمد قابل درى برغ است.

ئوهش حاضر محدوديتهايى دارد كه بايد در تفسير نتايج

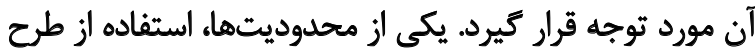

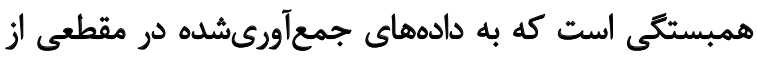

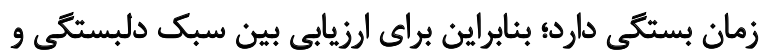

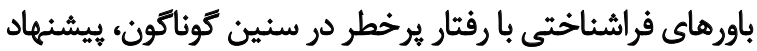

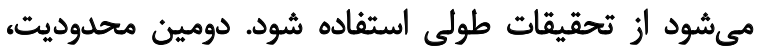

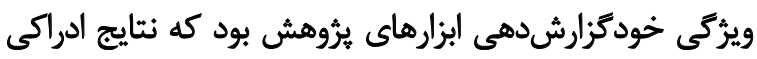

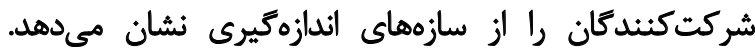

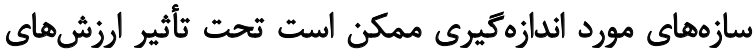

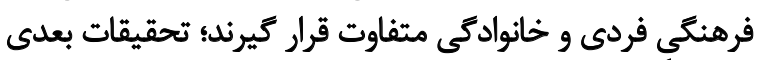

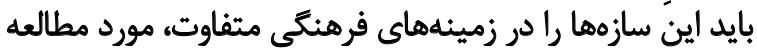

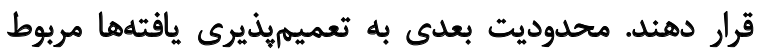

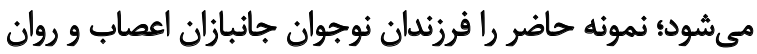

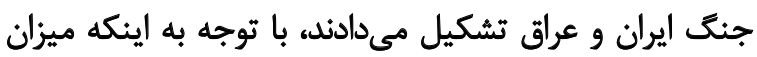

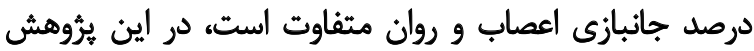

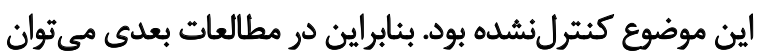

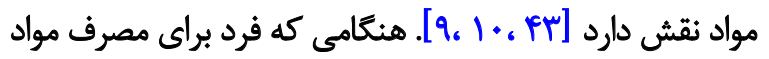

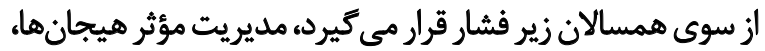

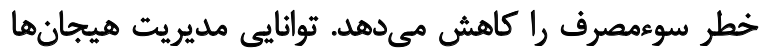

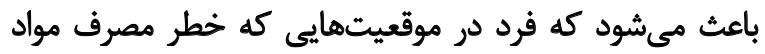

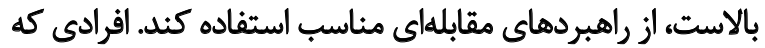

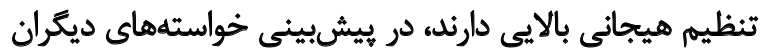

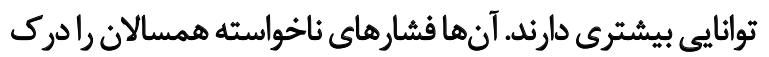
و هيجانهاي خود رابهتر مهار مي كنيند و درنتيجه در بر برابر مصرف مواد مقاومت بيشترى نشان مي دهند. به طور كلى بدتنظيمى هيجانى از آسيب يذيرى هيجانى ناشى

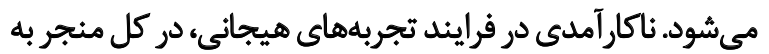

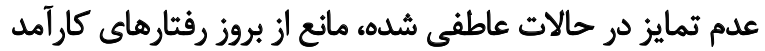

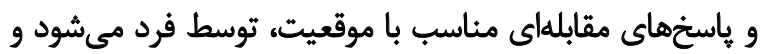

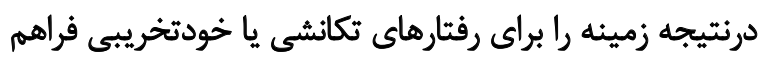

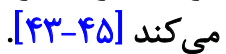

مشاهدات روزافزونى در برؤوهشها نشان مى دهد كه رفتارهاى

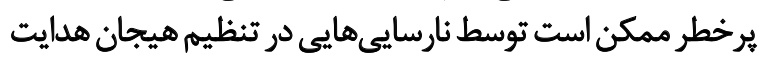

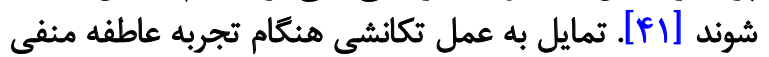

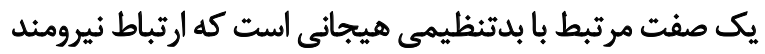

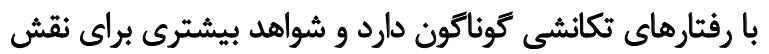

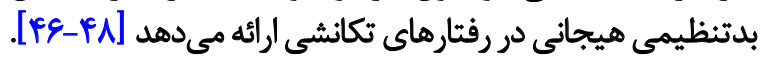

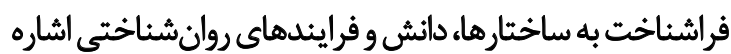

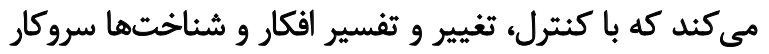

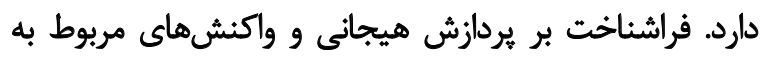

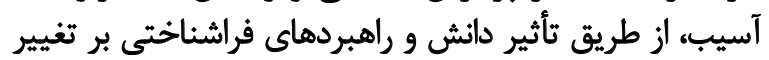

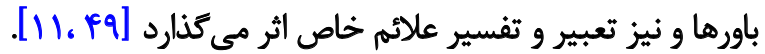

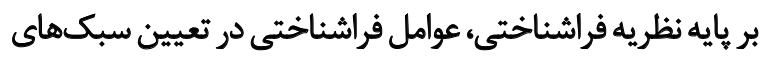

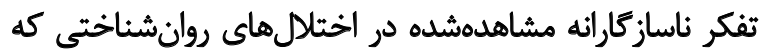

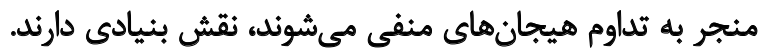

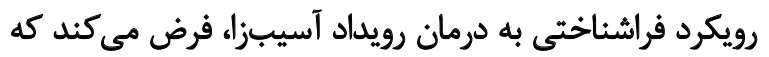

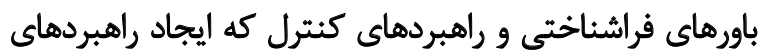

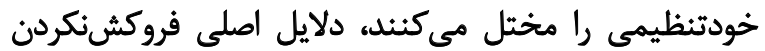
طبيعى علائم هستيند.

سندرم شناختى توجهى، شامل نوعى سبك تفكر تكرارشونده،

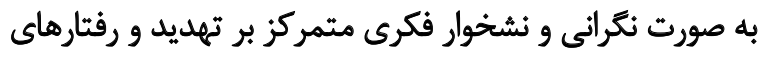

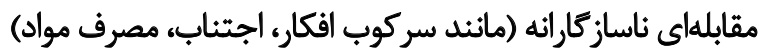

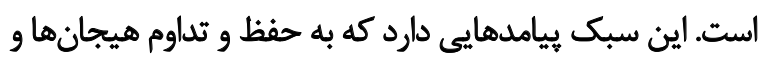

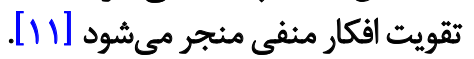

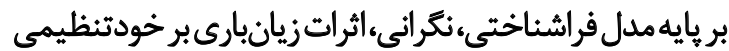

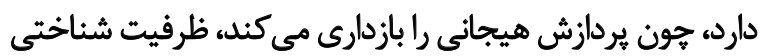

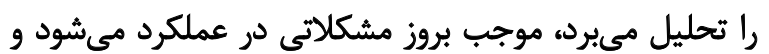
يردازش را بر تهديد متمركز مى كند. اين باور كه نترانى، مى مئواند 
نيز مسئولان بنياد شهيد و مدارس شاهد و ايثاركر كه در تكميل اين كار مساعدت خوبى با ما داشتند، صميمانه قدردانى مى كنيه.
با كنترل درصد جانبازى والدين كه بر يايه شدت علائم ماثم افراد

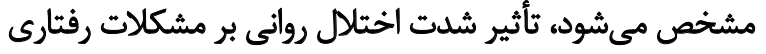

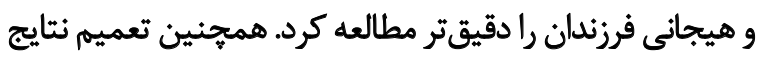

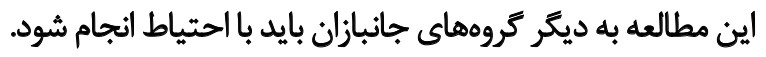

$$
\text { تئيجنيرى }
$$

با توجه به مطالب فوق، به نظر ميرسد نوجوانان در اينكونه

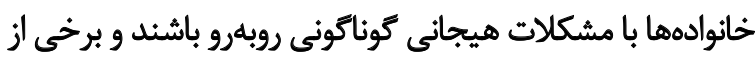

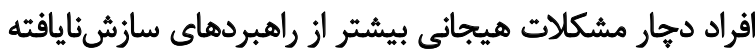

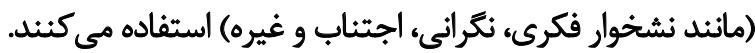

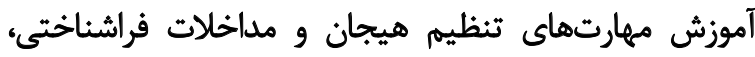

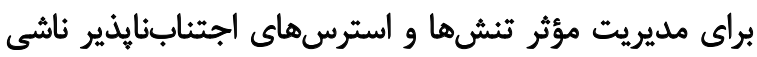

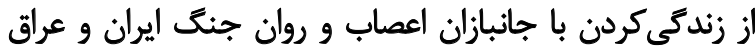

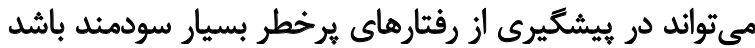

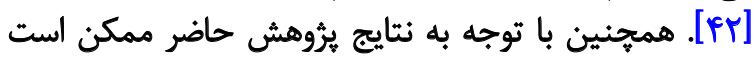

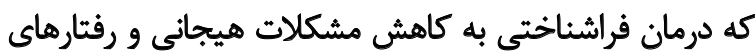

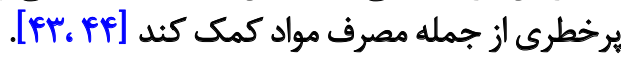

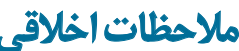

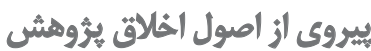

به كليه شركتكنيندكان در مورد اهداف مطالعه و همجنين

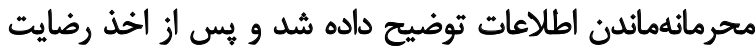

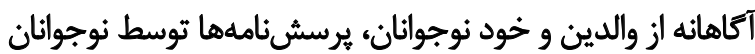

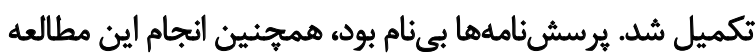
هيج هزينه و آسيبى براى نوجوانان نداشت. بئ.

$$
\text { حامي مالي }
$$

اين تحقيق هيج كونه كمك مالى خاصى از سازمانهاى تأمين

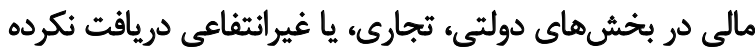

$$
\text { مشار كت نويسند مكّان }
$$

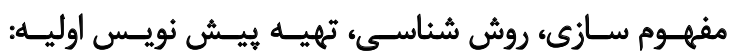

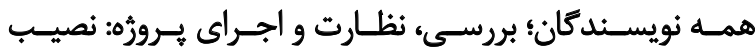

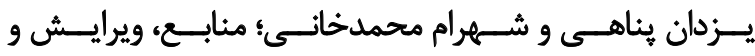

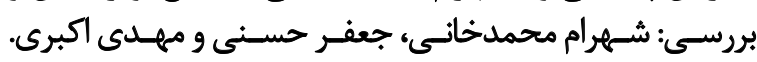

$$
\text { تعارض مناقع }
$$

بنابر اظهار نويسندكان، اين مقاله تعارض منافع ندارد.

$$
\text { تشكر و قدردانى }
$$

از همه نوجواناني كه در انجام اين يُروهش ما يارى رساندند و 


\section{References}

[1] Scharf M. Long-term effects of trauma: Psychosocial functioning of the second and third generation of Holocaust survivors. Development and Psychopathology. 2007; 19(2):603-22. [DOI:10.1017/ S0954579407070290] [PMID]

[2] Solomon Z, Debby-Aharon Sh, Zerach G, Horesh D. Marital adjustment, parental functioning, and emotional sharing in war Veterans. Journal of Family Issues. 2011; 32(1):127-47. [DOI:10.1177/0192513X10379203]

[3] Berger L. A psychological perspective of the holocaust. In: Braham RL, editor. Perspectives on the Holocaust, Holocaust Studies Series. Dordrecht: Springer; 1983. [DOI:10.1007/978-94015-6864-7_2]

[4] Shahrara M. [Developmental psychology (Persian)]. Tehran: Roshd Press; 2005.

[5] Mohammadkhani Sh. [Structural model of alcohol, tobacco and other drugs use among at risk adolescents: Direct and indirect impact of social and personal factors (Persian)]. Research in Psychological Health. 2007; 1(2):5-16.

[6] Mohammadkhani Sh. [Prevalence of cigarette smoking, alcohol drinking and illegal drugs use among Iranian adolescents (Persian)]. Journal of Kerman University of Medical Sciences. 2012; 19(1):32-48.

[7] Mohammadkhani Sh, Rezaee H. [The predictors of drug and alcohol abuse among adolescents (Persian)]. Journal of Torbat Heydariyeh University of Medical Sciences. 2015; 2(4):1-9.

[8] Qahvehchi F, Mohammadkhani Sh. [The correlation between metacognitive beliefs, coping strategies, and negative emotions with students' smoking (Persian)]. Journal of Advances in Medical and Biomedical Research. 2012; 20(81):93-102.

[9] Ramezanzadeh F, Moradi AR, Mohammadkhani Sh. [Effectiveness training emotion regulation skills in executive function and emotion regulation strategies of adolescents at risk (Persian)]. Journal of Cognitive Psychology. 2014; 2(2):37-45

[10] Mohammadkhani Sh, Rezaee Jamaloei H. [Relationship between cigarette and hookah smoking with individual, family and social factors in adolescents (Persian)]. Journal of Sabzevar University of Medical Sciences. 2016; 23(2):262-80.

[11] Wells A. Metacognitive therapy for anxiety and depression. New York: Guilford Press; 2009.

[12] Marlatt GA, Gordon JR. Relapse Prevention: Maintenance strategies in addictive behaviour change. New York, NY: Guilford; 1985.

[13] Marlatt GA, Witkiewitz K. Relapse prevention for alcohol and drug problem. In: Alan Marlatt G, Donovan DM, editors. Relapse Prevention: Maintenance Strategies in the Treatment of Addictive Behaviors. New York, NY: Guilford Press; 2005.

[14] Spada MM, Caselli G, Wells A. A triphasic metacognitive formulation of problem drinking. Clinical Psychology \& Psychotherapy. 2013; 20(6):494-500. [DOI:10.1002/cpp.1791] [PMID]

[15] Toneatto T. Metacognition and substance use. Addictive Behaviors. 1999; 24(2):167-74. [DOI:10.1016/S0306-4603(98)00126-9]

[16] Mazloom M, Yaghubi H, Mohammadkhani Sh. [The relationship of metacognitive beliefs and emotion regulation difficulties with post traumatic stress disorder (Persian)]. International Journal of Behavioral Sciences. 2014; 8(2):105-13.

[17] Oshri A, Sutton TE, Clay-Warner J, Miller JD. Child maltreatment types and risk behaviors: Associations with attachment style and emotion regulation dimensions. Personality and Individual Differences. 2015; 73:127-33. [DOI:10.1016/j.paid.2014.09.015]

[18] Riggs SA, Riggs DS. Risk and resilience in military families experiencing deployment: The role of the family attachment network. Journal of Family Psychology. 2011; 25(5):675-87. [DOI:10.1037/a0025286] [PMID]

[19] Dubois-Comtois K, Cyr C, Pascuzzo K, Lessard M, Poulin C. Attachment theory in clinical work with adolescents. Journal of Child \& Adolescent Behavior. 2013; 1(3):1000111.

[20] Waters SF, Virmani EA, Thompson RA, Meyer S, Abigail Raikes H, Jochem R. Emotion regulation and attachment: Unpacking two constructs and their association. Journal of Psychopathology and Behavioral Assessment. 2010; 32(1):37-47. [DOI:10.1007/ s10862-009-9163-z] [PMID] [PMCID]

[21] Garnefski N, Kraaij V, Spinhoven P. Negative life events, cognitive emotion regulation and emotional problems. Personality and Individual Differences. 2001; 30(8):1311-27. [DOI:10.1016/S01918869(00)00113-6]

[22] Hasani J. [The reliability and validity of the short form of the cognitive emotion regulation questionnaire (Persian)]. Journal of Research in Behavioural Sciences. 2011; 9(4):229-40.

[23] Bacow TL, Pincus DB, Ehrenreich JT, Brody LR. the metacognitions questionnaire for children: Development and validation in a clinical sample of children and adolescents with anxiety disorders. Journal of Anxiety Disorders. 2009; 23(6):727-36. [DOI:10.1016/j.janxdis.2009.02.013] [PMID]

[24] Ghaderi B, Mohammadkhani Sh, Hassanabadi HR. [Cognitive and metacognitive predictors of anxiety in adolescents (Persian)] Journal of Clinical Psychology. 2016; 7(4):13-26.

[25] Mohamadkhani Sh. [Psychological empowerment program - the school community: Health promotion with a focus on life skills training to at-risk students (Persian)]. Iran: United Nations Office on Drugs and Crime (UNODC) in Iran and Drug Control Headquarters. UNODC; 2012.

[26] Mohamadkhani Sh. [Primary prevention of addiction, with a focus on school (Persian)]. Iran: Drug Control Headquarters. 2012b. UNODC; 2012.

[27] Mohamadkhani Sh. [Strategies for the prevention of drug abuse among students at risk identification, assessment and intervention (Persian)]. Iran: United Nations Office on Drugs and Crime (UNODC) in Iran and Drug Control Headquarters. UNODC; 2014

[28] Collins NL, Read SJ. Cognitive representations of attachment: The structure and function of working Models. In: Bartholomew K, Perlman D. Advances in Personal Relationships, Volume 5. London: Jessica Kingseley; 1994.

[29] Mohammadi K, Samavi A, Ghazavi Z. The relationship between attachment styles and lifestyle with marital satisfaction. Iranian Red Crescent Medical Journal. 2016; 18(4):e23839. [DOI:10.5812/ircmj.23839] [PMID] [PMCID]

[30] Kalantari Kh. [Structural equation modeling in socio - Anderson JC, Gerbing DW. Structural equation modeling in practice: A 
review and recommended two-step approach. Psychological Bulletin. 1988; 103(3):411-23. [DOI:10.1037/0033-2909.103.3.411]

[31] Hooman HA. [Structural equation modeling with Lisrel application (Persian)]. Tehran: Samt; 2009.

[32] Taft CT, Pless AP, Stalans LJ, Koenen KC, King LA, King DW. Risk factors for partner violence among national sample of combat veterans. Journal of Consulting and Clinical Psychology. 2005; 73(1):151-9. [DOI:10.1037/0022-006X.73.1.151] [PMID]

[33] Allen ES, Rhoades GK, Stanley SM, Markman HJ. Hitting home: Relationships between recent deployment, posttraumatic stress symptoms, and marital functioning for army couples. Journal of Family Psychology. 2010; 24(3):280-8. [DOI:10.1037/ a0019405] [PMID] [PMCID]

[34] Renshaw KD, Rodrigues CS, Jones DH. Psychological symptoms and marital satisfaction in spouses of Operation Iraqi Freedom veterans: Relationships with spouses' perception of veterans' experiences and symptoms. Journal of Family Psychology. 2008; 22(4):586-94. [DOI:10.1037/0893-3200.22.3.586] [PMID]

[35] Maršanić VB, Margetić BA, Zečević I, Herceg M. The prevalence and psychosocial correlates of suicide attempts among inpatient adolescent offspring of croatian PTSD male war veterans. Child Psychiatry \& Human Development. 2014; 45(5):577-87. [DOI:10.1007/s10578-013-0426-2] [PMID]

[36] Klarić M, Frančišković T, Klarić B, Kvesić A, Kaštelan A, Graovac $\mathrm{M}$, et al. Psychological problems in children of war veterans with posttraumatic stress disorder in Bosnia and Herzegovina: Cross-sectional study. Croatian Medical Journal. 2008; 49(4):4918. [DOI:10.3325/cmj.2008.4.491] [PMID] [PMCID]

[37] Ellis BJ, Dishion TJ, Gray P, Hawley PH, Volk AA, Giudice MD, et al. The evolutionary basis of risky adolescent behavior: Implications for science, policy, and practice. Developmental Psychology. 2012; 48(3):598-623. [DOI:10.1037/a0026220] [PMID]

[38] Shaver PR, Mikulincer M. Adult attachment strategies and the regulation of emotion. In: Gross JJ, editor. Handbook of Emotion Regulation. New York: Guilford Press; 2007.

[39] Mikulincer M, Shaver PR. The attachment behavioral system in adulthood: Activation, psychodynamics, and interpersonal processes. In: Contributors. Advances in Experimental Social Psychology. Vol. 35. 2003. [DOI:10.1016/S0065-2601(03)01002-5]

[40] Weiss NH, Sullivan TP, Tull MT. Explicating the role of emotion dysregulation in risky behaviors: A review and synthesis of the literature with directions for future research and clinical practice. Current Opinion in Psychology. 2015; 3:22-9. [DOI:10.1016/j. copsyc.2015.01.013] [PMID] [PMCID]

[41] Parker JDA, Taylor RN, Eastabrook JM, Schell SL, Wood LM. Problem gambling in adolescence: Relationships with internet misuse, gaming abuse and emotional intelligence. Personality and Individual Differences. 2008; 45(2):174-80. [DOI:10.1016/j. paid.2008.03.018]

[42] Trinidad DR, Johnson CA. The association between emotional intelligence and early adolescent tobacco and alcohol use. Personality and Individual Differences. 2002; 32(1):95-105. [DOI:10.1016/ S0191-8869(01)00008-3]

[43] Jacobson CM, Muehlenkamp JJ, Miller AL, Turner JB. Psychiatric impairment among adolescents engaging in different types of deliberate self- harm. Journal of Clinical Child \& Adolescent Psychology. 2008; 37(2):363-75. [DOI:10.1080/15374410801955771] [PMID]
[44] Linehan MM, Bohus M, Lynch TR. Dialectical behavior therapy for pervasive emotion dysregulation. In: Gross JJ, editor. Handbook of Emotion Regulation. New York: Guilford Press; 2007.

[45] Azimi M, Nori R, Mohammadkhani Sh. [Comparison of cognitive emotion regulation, general health, problem-solving styles and academic performance of adolescent girls with addicted and nonaddicted parents (Persian)]. Journal of Research in Psychological Health. 2016; 10(3):38-48.

[46] Shaver PR, Mikulincer M. Attachment-related psychodynamics. Attachment \& Human Development. 2002; 4(2):133-61. [DOI:10.1080/14616730210154171] [PMID]

[47] Weiss NH, Tull MT, Viana AG, Anestis MD, Gratz KL. Impulsive behaviors as an emotion regulation strategy: Examining associations between PTSD, emotion dysregulation, and impulsive behaviors among substance dependent inpatients. Journal of Anxiety Disorders. 2012; 26(3):453-8. [DOI:10.1016/j. janxdis.2012.01.007] [PMID] [PMCID]

[48] Corcocan KM, Segal ZV. Metacognition in depressive and anxiety disorders: Current disorders. International Journal of Cognitive Therapy. 2008; 1(1):33-44. [DOI:10.1521/ijct.2008.1.1.33]

[49] Spada MM, Wells A. Metacognitions across the continuum of drinking behaviour. Personality and Individual Differences. 2010; 49(5):425-9. [DOI:10.1016/j.paid.2010.04.011]

[50] Spada M, Wells A. A metacognitive model of problem drinking. Clinical Psychology \& Psychotherapy. 2009; 16(5):383-93. [DOI:10.1002/cpp.620] [PMID]

[51] Dansby VS, Marinelli RP. Adolescent children of Vietnam combat veteran fathers: A population at risk. Journal of Adolescence. 1999; 22(3):329-40. [DOI:10.1006/jado.1999.0225] [PMID]

[52] Noorbala AA. [Psychiatric disoders ib combatants (Persian)] Journal of Military Medicine. 2003; 4(4):257-60. 
This Page Intentionally Left Blank 